\title{
Tadpole-induced electroweak symmetry breaking and pNGB Higgs models
}

\author{
Roni Harnik, Kiel Howe and John Kearney \\ Theoretical Physics Department, Fermi National Accelerator Laboratory, \\ Batavia, IL 60510 U.S.A. \\ E-mail: roni@fnal.gov, khowe@fnal.gov, jkearney@fnal.gov
}

ABSTRACT: We investigate induced electroweak symmetry breaking (EWSB) in models in which the Higgs is a pseudo-Nambu-Goldstone boson (pNGB). In pNGB Higgs models, Higgs properties and precision electroweak measurements imply a hierarchy between the EWSB and global symmetry-breaking scales, $v_{H} \ll f_{H}$. When the pNGB potential is generated radiatively, this hierarchy requires fine-tuning to a degree of at least $\sim v_{H}^{2} / f_{H}^{2}$. We show that if Higgs EWSB is induced by a tadpole arising from an auxiliary sector at scale $f_{\Sigma} \ll v_{H}$, this tuning is significantly ameliorated or can even be removed. We present explicit examples both in Composite Higgs models based on $\mathrm{SO}(5) / \mathrm{SO}(4)$ and in Twin Higgs models. For the Twin case, the result is a fully natural model with $f_{H} \sim 1 \mathrm{TeV}$ and the lightest colored top partners at $2 \mathrm{TeV}$. These models also have an appealing mechanism to generate the scales of the auxiliary sector and Higgs EWSB directly from the scale $f_{H}$, with a natural hierarchy $f_{\Sigma} \ll v_{H} \ll f_{H} \sim \mathrm{TeV}$. The framework predicts modified Higgs coupling as well as new Higgs and vector states at LHC13.

Keywords: Beyond Standard Model, Higgs Physics, Technicolor and Composite Models ARXIV EPRINT: 1603.03772 


\section{Contents}

1 Introduction 1

$2 \mathrm{SO}(5) / \mathrm{SO}(4) \mathrm{MCHM}$ model $\quad 4$

2.1 MCHM with a tadpole 5

2.2 Radiative tuning from the top sector 5

2.3 Dynamical auxiliary sectors 8

$\begin{array}{lll}2.4 \text { UV considerations } & 11\end{array}$

3 Twin Higgs model 11

$\begin{array}{ll}3.1 \text { Twin Higgs with a tadpole } & 12\end{array}$

$\begin{array}{lll}3.2 & \text { Radiative tuning } & 14\end{array}$

$\begin{array}{lll}3.3 & \text { Dynamical twin auxiliary sector } & 16\end{array}$

$\begin{array}{ll}3.4 \text { UV issues } & 18\end{array}$

4 Experimental constraints $\quad 18$

5 Conclusion $\quad 22$

A Expressions for two-site models $\quad 23$

$\begin{array}{lll}\text { A. } 1 & 5+1 & 23\end{array}$

$\begin{array}{lll}\text { A. } 2 & 8+1 & 24\end{array}$

B Strongly-coupled auxiliary sectors $\quad 24$

B.1 Composite Higgs 24

B.2 Twin Higgs 26

\section{Introduction}

The discovery of the Higgs boson has sharpened the problem of the naturalness of the electroweak (EW) scale. An attractive solution is that the Higgs boson is a composite pseudo-Nambu-Goldstone Boson (pNGB) of a global symmetry that is spontaneously broken at a scale $f_{H}$ not far above the electroweak scale $v_{H}=246 \mathrm{GeV}[1,2]$. More modern realizations of this idea include Composite Higgs $(\mathrm{CH})$ models (with partial compositeness) [3-5], as well as Twin Higgs (TH) [6, 7] and Little Higgs [8-11].

Standard Model (SM) interactions must explicitly break the global symmetries protecting the pNGB Higgs. This results in radiative contributions to the pNGB potential, with the largest contributions arising from the top Yukawa coupling and the gauging of $\mathrm{SU}(2)_{L}$, and in minimal composite Higgs models the pNGB potential is entirely generated by these contributions. As such, the mass scales of new top and gauge partners restoring 
the global symmetries are connected to that of the Higgs boson. For instance, the contributions from the top sector perturb the vev and physical Higgs mass proportionally by an amount of size

$$
\left|\delta m_{h}^{2}\right| \gtrsim \frac{3 y_{t}^{2}}{4 \pi^{2}} m_{*}^{2} \sim(125 \mathrm{GeV})^{2}\left(\frac{m_{*}}{500 \mathrm{GeV}}\right)^{2}
$$

where $m_{*}$ is the mass scale of the top partners which restore the global symmetry. If these resonances are sufficiently light, the physical Higgs mass $m_{h}=125 \mathrm{GeV}$ can be obtained naturally without any tuning. Direct experimental limits on the scale $m_{*}$ of top partners [12-14] give lower bounds on the tuning of such theories, but current limits permit a totally natural mass scale for the Higgs when colored top partner decays are hidden $[15,16]$ or the global symmetry is partially restored by neutral particles, as in Twin Higgs models [6, 7].

However, observations of Higgs properties [17-19] require $v_{H} \ll f_{H}$ so that the curvature of the pNGB manifold does not induce significant Higgs coupling deviations from the SM values (see, e.g., [20, 21]). SM-like Higgs measurements at the level of $\sim 10 \%$ constrain $\frac{f_{H}^{2}}{v_{H}^{2}} \gtrsim 10$, and future measurements will reach the $\sim 1 \%$ level [22-24]. This makes realizing a natural model much more difficult - composite Higgs models with top partners in minimal representations (e.g., $\mathrm{MCHM}_{5}, \mathrm{MCHM}_{5+1}$ ) can only obtain $m_{h}=125 \mathrm{GeV}$ when $v_{H} \ll f_{H}$ with severe radiative tuning [20, 21]. Extended top partner sectors (e.g., $\mathrm{MCHM}_{14+1}$ ) can improve the situation, but the structure of radiative contributions to the pNGB potential still leads to an 'irreducible' tuning $\Delta \gtrsim \frac{f_{H}^{2}}{2 v_{H}^{2}}$.

These obstacles motivate studying pNGB Higgs models with a combination of additional tree-level contributions to the potential and top sectors that minimize radiative contributions, as such models stand the best chance to be 'maximally natural'. One wellknown strategy, used in Little Higgs (as well as some TH models [25]), is to introduce additional dynamics generating a tree-level quartic without a significant contribution to the Higgs mass-squared parameter. So, small radiatve contributions to the potential allow a natural hierarchy $v_{H} \ll f_{H}$, while the dominant quartic term raises the Higgs mass to the observed value.

Here, we study an alternative approach. The pNGB potential will naturally be of the size of the radiative contributions, but with a positive mass-squared stabilizing the vacuum at $v_{H}=0$. An auxiliary decoupling EWSB sector $\Sigma$ is then introduced to trigger Higgs EWSB through a linear coupling to the Higgs sector, perturbing the Higgs vacuum to a non-zero vev with a natural hierarchy $f_{\Sigma} \ll v_{H} \ll f_{H}$ (where the total scale of EWSB is $v^{2}=f_{\Sigma}^{2}+v_{H}^{2}$ ). This is an application of Bosonic Technicolor (BTC) or, as it is more recently dubbed, induced EWSB [26-42] to a pNGB Higgs. A schematic comparison of this approach to the tuned minimal radiative approach is shown in figure 1.

The possibility of triggering EWSB by a tadpole leads to modified Higgs phenomenology and to new Higgs-like states. The fact that this is a viable option post Higgs discovery is non-trivial and was shown in [39-41]. Note that this mechanism has qualitatively different features compared to models where the pNGB mixes with an inert auxiliary doublet [43].

When the auxiliary sector is weakly coupled, there is an energy regime where the pNGB Higgs and auxiliary sector are well-described by a two-Higgs doublet model (2HDM) 

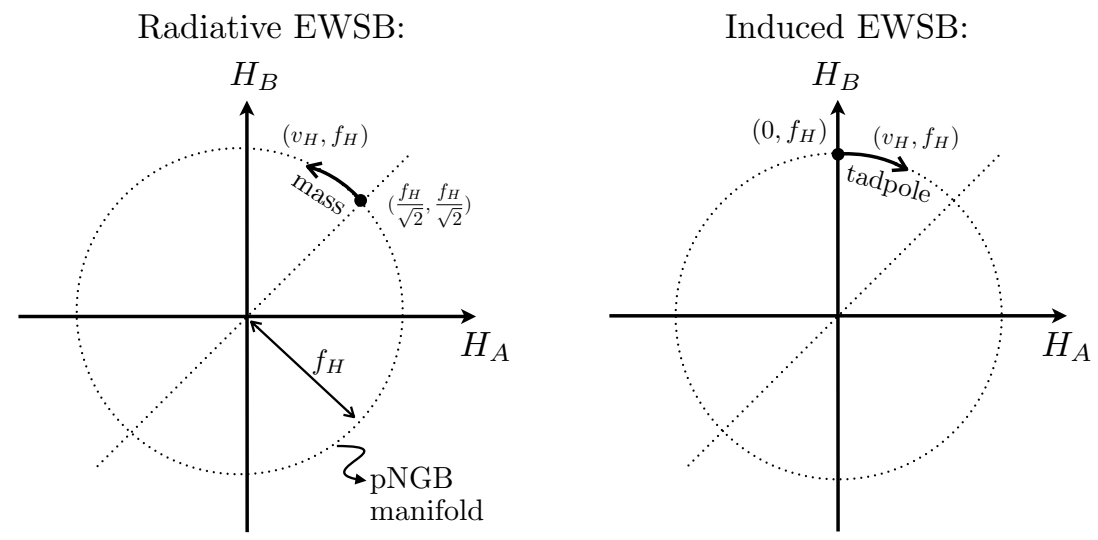

Figure 1. A schematic depiction of "regular" radiative EWSB (left) versus induced EWSB (right) in a pNGB Higgs model. In this figure we take the Twin Higgs as an example where $H_{A}$ is the SM Higgs doublet and $H_{B}$ is its mirror partner (but the mechanism applies more broadly). In both cases the non-linear sigma model constrains the vev to live on a "pNGB manifold" (dotted circle). In the radiative EWSB the generic, untuned, EW vev is tuned down from $f_{H}$ to $v_{H}$ using a mass term. In the induced case an untuned $\mathrm{EW}$ vev of zero is brought up to $v_{H}$ without tuning by a tadpole.

(see, e.g., [44]). While we will find this limit useful to illustrate the basic features of the model, the most interesting parts of parameter space correspond to a strongly coupled (for example, technicolor-like) auxiliary sector that is not well-described by a weakly coupled 2HDM. A naïve strong-coupling analysis will allow us to understand the effects on the pNGB potential in this case.

The tuning problem in pNGB models in many ways resembles the little hierarchy problem of the minimal supersymmetric standard model (MSSM), where obtaining $m_{h}=$ $125 \mathrm{GeV}$ radiatively requires stop masses $m_{\tilde{t}} \gg \mathrm{TeV}$ and/or large A-terms, both of which directly contribute to the tuning [45]. It is not surprising then that parallels can be drawn between proposed solutions in the two frameworks. For example, the addition of radiatively-safe tree-level quartics is commonplace both in supersymmetric models (as in, e.g., the NMSSM) [46-49] and in Little Higgs. Indeed, the approach we take here to reconciling the Higgs mass with naturalness has been considered previously in the context of supersymmetric models [27, 28, 36-42], but has not yet been employed in composite/pNGB Higgs models.

In the subsequent sections, we will study models where a composite Higgs sector is coupled to an extra tadpole EWSB sector. For the composite Higgs sector we focus on two well-studied possibilities: section 2 studies the coupling of a tadpole sector to a 'conventional' Minimal Composite Higgs models (MCHM) based on $\mathrm{SO}(5) / \mathrm{SO}(4)$ [5, 20, 21], and section 3 extends this analysis for composite Twin Higgs models $[6,7,50,51]$ based on $\mathrm{SO}(8) / \mathrm{SO}(7)$ (or $\mathrm{SU}(4) / \mathrm{SU}(3)$ for weakly-coupled UV completions). Twin Higgs models are of particular interest because the 'irreducible' $\frac{f_{H}^{2}}{2 v_{H}^{2}}$ can be completely removed giving, for example, an untuned model with a global symmetry-breaking scale $f_{H} \sim 1 \mathrm{TeV}$ and colored top partners at $2 \mathrm{TeV}$. 
In each case, we study first the limit of a single "frozen" linear tadpole operator in the composite Higgs sector, disregarding the dynamics of the the tadpole sector. In this approximation, we show that the tuning in the Higgs sector can be significantly ameliorated. We then consider the full dynamics of the tadpole sector, and show that the "frozen" tadpole approximation is a good description so long as the tadpole sector itself is strongly coupled. In section 4 we discuss phenomenological constraints on the Higgs properties, extended Higgs or $\Sigma$ sector states, and top partners for both the MCHM and Twin Higgs cases. Finally, we conclude in section 5.

\section{$2 \mathrm{SO}(5) / \mathrm{SO}(4) \mathrm{MCHM}$ model}

In Composite Higgs models, the SM Higgs is identified with a pNGB in the coset $G / H$ of the spontaneously-broken global symmetry $G \rightarrow H$. For the $\mathrm{SO}(5) / \mathrm{SO}(4)$ models relevant to our discussion, the radiatively-generated Higgs potential can be parameterized as [20]

$$
V(h)=\alpha f_{H}^{4} \sin ^{2}\left(\frac{h}{f_{H}}\right)+\beta f_{H}^{4} \sin ^{4}\left(\frac{h}{f_{H}}\right)
$$

where $f_{H}$ is the scale of spontaneous $G$ breaking. For $\alpha<0$, EWSB with scale $v_{H}$ in the Higgs sector is triggered. The hierarchy compared to the global symmetry breaking scale is

$$
\frac{v_{H}^{2}}{f_{H}^{2}}=\sin ^{2}\left(\frac{\langle h\rangle}{f_{H}}\right)=-\frac{\alpha}{2 \beta},
$$

while the physical mass-squared is

$$
m_{h}^{2}=2 \beta f_{H}^{2} \sin ^{2}\left(\frac{2\langle h\rangle}{f_{H}}\right)=-4 \alpha f_{H}^{2} \cos ^{2}\left(\frac{\langle h\rangle}{f_{H}}\right)
$$

and $m_{h}=125 \mathrm{GeV}$ is realized for $\beta=\beta_{\mathrm{SM}} \simeq 1 / 32$.

A key point is that radiative contributions to the potential from the explicit $G$-breaking couplings of the SM generically generate $|\alpha| \gtrsim \beta$. To realize a hierarchy $v_{H} \ll f_{H}$ requires $|\alpha| \ll \beta$, and in the case of a purely radiative potential this can only be arranged with a tuned cancellation among the different contributions to $\alpha$. Explicitly, assuming that the physics responsible for generating the required $\beta=\beta_{\mathrm{SM}}$ also generates a comparable contribution to $\alpha$, and taking $\frac{v_{H}^{2}}{f_{H}^{2}} \ll 1$, implies a tuning

$$
\frac{\delta \alpha}{\alpha} \gtrsim \frac{\beta}{2 \beta\left(v_{H}^{2} / f_{H}^{2}\right)}=\frac{f_{H}^{2}}{2 v_{H}^{2}} .
$$

However, this tuning is not 'irreducible' - it can be avoided by including an additional tadpole-like contribution to the potential. The structure of the low-energy theory is that of 'induced' EWSB [41, 42]. In induced EWSB, the SM-like Higgs vev arises as a result of a coupling linear in the Higgs to a tadpole sector parameterized by an $\mathrm{SU}(2)$ doublet scalar $\Sigma$ that breaks the electroweak symmetry with $\langle|\Sigma|\rangle=\frac{f_{\Sigma}}{\sqrt{2}}$,

$$
V(H) \supset-\mu^{2} H \cdot \Sigma+\text { h.c. }
$$


If this additional sector were not present or did not acquire a vev, Higgs EWSB would not occur. Induced EWSB is the limit that the extra heavy modes arising primarily from the $\Sigma$ sector are decoupled, so that the dominant effect on low energy states can be viewed as arising from an effective tadpole for the Higgs. We first focus on this limit in section 2.1, and we show in section 2.2 that for some concrete choices of a top partner sector the tuning is substantially improved. We return to the dynamics of the tadpole sector in section 2.3 to show that the leading effects in realistic models are captured by the decoupling limit analysis and describe some of the properties of the new electroweak states. In section 2.4, we discuss a few additional considerations that should be taken into account when considering potential UV completions of this type of model.

\subsection{MCHM with a tadpole}

For a composite Higgs model, we can parameterize the tadpole eq. (2.5) by a term in the non-linear realization $\gamma=\mu^{2} f_{\Sigma} / f_{H}^{3}$,

$$
V(h)=-\gamma f_{H}^{4} \sin \left(\frac{h}{f_{H}}\right)+\alpha f_{H}^{4} \sin ^{2}\left(\frac{h}{f_{H}}\right)+\ldots
$$

such that

$$
\frac{v_{H}}{f_{H}}=\sin \left(\frac{\langle h\rangle}{f_{H}}\right)=\frac{\gamma}{2 \alpha}, \quad m_{h}^{2}=2 \alpha\left(f_{H}^{2}-v_{H}^{2}\right) .
$$

This mechanism requires $\alpha>0$, such that $v_{H}=0$ for $\gamma=0$. The tadpole perturbs the vacuum from $v_{H}=0$ and a small value of $\gamma$ naturally leads to $v_{H} \ll f_{H}$. As such, the correct Higgs mass and vev can be achieved even with $\beta \ll \beta_{\mathrm{SM}}$. Moreover, the tadpole breaks an otherwise exact global $Z_{2} \subset \mathrm{SU}(2)_{\Sigma}$ under which $\Sigma \rightarrow-\Sigma$, so $\gamma \ll 1$ is technically natural. As long as radiative contributions to the mass-squared can be made naturally small, $\delta \alpha f_{H}^{2} \lesssim m_{h}^{2}$, the overall naturalness of the model can be improved.

\subsection{Radiative tuning from the top sector}

To realize the top Yukawa coupling, the top sector of a composite Higgs model must break the global symmetries, giving a contribution to the pNGB potential quadratically sensitive to the top partner masses $m_{*}$. Due to strong limits on top partners, this is typically the most significant contribution to $\delta \alpha$. In the partial compositeness framework, the mixings of the elementary states and composite top partners generate the top Yukawa coupling [5, 20, 21], and there is a rough lower limit on the top partner mass scale $m_{*}$ compared to the global symmetry breaking scale, $m_{*} \gtrsim \frac{y_{t} f_{H}}{\sqrt{2}}$. This translates to a lower bound on the tuning,

$$
\frac{\delta \alpha}{\alpha_{\mathrm{obs}}} \gtrsim \frac{3 y_{t}^{4} f_{H}^{2}}{8 \pi^{2}\left(\frac{v_{H}^{2}}{4}\right)} \simeq \frac{1}{6}\left(\frac{f_{H}^{2}}{2 v_{H}^{2}}\right) .
$$

This limit is weaker than the 'irreducible' tuning eq. (2.4) in a purely radiative potential, but applies more generally. However, for a fixed value of $f_{H}$, alternatives to the purely radiative composite Higgs potential, including the tadpole structure considered here, have the potential to improve the tuning by a factor of $\sim 6$. Moreover, this improvement can be even larger for top sectors that do not saturate eq. (2.4), as in the example considered here. 
The exact form of the radiative Higgs potential is determined by the embedding of the top partners in the global symmetry group. In this section we study explicitly the $\mathrm{MCHM}_{5+1}$ model coupled to a tadpole sector. This model has composite fermionic partners (including colored vector-like top partners) in the $5=4+1, \psi=\left(\psi_{4}^{i}, \psi_{1}\right)$ and $\psi^{c}=$ $\left(\psi_{4}^{c i}, \psi_{1}^{c}\right)[5,20,21]$, with $q_{L}$ mixing as a $5=4+1$ and $t_{R}$ as a singlet. For this embedding, only the mixings of $q_{L}$ explicitly break the global symmetry. While in principal eq. (2.8) may be realized for some choice of MCHM top sector, for the $\mathrm{MCHM}_{5+1}$ case contributions to $\alpha$ are quadratically sensitive to the top partner mass while contributions to $\beta$ are only logarithmically sensitive, yielding $|\alpha| \sim \frac{m_{*}^{2}}{f_{H}^{2}}|\beta|$. As a result, we find that the radiative tuning is always worse than $\sim f_{H}^{2} / 2 v_{H}^{2}$. Nonetheless, it is possible to achieve a substantial improvement over the $\sim 1 \%$ tuning of the $\mathrm{MCHM}_{5+1}$ model in the absence of a tadpole sector. As such, $\mathrm{MCHM}_{5+1}$ is an interesting example of a model with a minimal top sector in which tuning can be greatly reduced by induced EWSB. In section 3 we will consider Twin Higgs as an example of a model that can in fact saturate eq. (2.8), potentially allowing a fully natural model.

Motivated by bounds on Higgs properties, we will fix $f_{H}=1 \mathrm{TeV}\left(\frac{f_{H}^{2}}{v_{H}^{2}} \simeq 16\right)$ as a benchmark in this section with $f_{\Sigma}=70 \mathrm{GeV}$ (giving $v_{H}=236 \mathrm{GeV}$ ). As discussed in greater detail in section 4 , this benchmark is at the edge of current limits. For different values of $f_{H}$, the top partner masses can be scaled as $f_{H}$ and the associated tuning as $f_{H}^{2}$; the tuning from the top sector is insensitive to $f_{\Sigma}$ when $f_{\Sigma} \ll v_{H}$. We require (to leading order in $\left.\frac{v_{H}^{2}}{f_{H}^{2}}\right)$,

$$
\alpha_{\mathrm{obs}}=\alpha_{0}+\delta \alpha \simeq \frac{(125 \mathrm{GeV})^{2}}{2 f_{H}^{2}} \simeq \frac{1}{8} \frac{v_{H}^{2}}{f_{H}^{2}}
$$

to realize EWSB with the observed Higgs mass. We can therefore estimate the tuning of the tadpole model

$$
\Delta=\frac{\partial \ln \alpha}{\partial \ln \alpha_{0}}=1-\frac{\delta \alpha}{\alpha_{\mathrm{obs}}}
$$

The radiative contribution $\delta \alpha$ from the top sector is often negative in the concrete models of the top sector we study. However, as discussed in section 2.4, the $\alpha_{0}>0$ required for induced EWSB can easily be generated by gauge contributions to the pNGB potential to tune $\alpha=\alpha_{\text {obs }}>0$.

In a two-site model $[52,53]$ for this composite sector, the radiative contributions to the Higgs potential can be calculated directly and parameterized in terms of two top partner mass scales, $m_{1}$ and $m_{4}$, and the mixing angles $\sin \theta_{L, R}$ of the top quark with the composites $\psi_{A}, \psi_{A}^{c}$. To leading order in $\frac{v_{H}}{f_{H}}$, the $\mathrm{SU}(2)_{L}$-doublet top partners have masses $m_{4}$ and $M_{4}=m_{4} / \cos \theta_{L}$, and the $\mathrm{SU}(2)_{L}$-singlet top partner has mass $M_{1}=m_{1} / \cos \theta_{R}$. The Yukawa coupling is

$$
y_{t}=\frac{m_{4}}{f_{H}} \sin \theta_{L} \sin \theta_{R}
$$

to leading order, which requires $m_{4} \gtrsim f_{H}$, and gives a lower bound $\frac{M_{4}}{f_{H}} \gtrsim \frac{2}{\sin \theta_{R}}$ for the top partner mixing with the elementary $t_{L}$. For numerical results, we use $y_{t}=y_{t, \mathrm{SM}}\left(v / v_{H}\right)$, where $y_{t, \mathrm{SM}}$ is the $\overline{\mathrm{MS}}$ value at $1 \mathrm{TeV}$. The full definition of the two-site model and the 


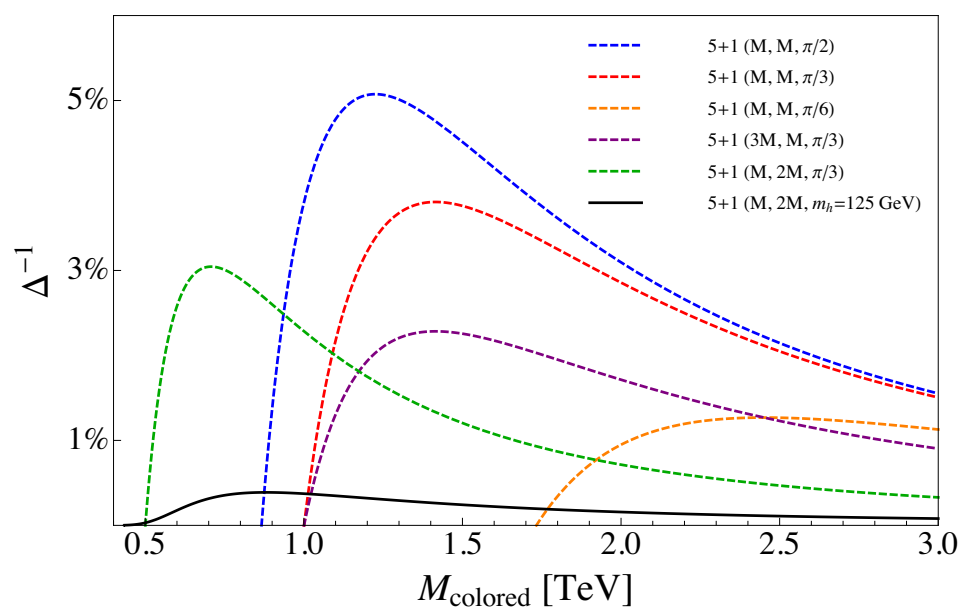

Figure 2. Top sector radiative tuning in the $\mathrm{SO}(5) / \mathrm{SO}(4) 5+1$ model (or $\mathrm{MCHM}_{5+1}$ ) with a tadpole as a function of the lightest colored top partner mass $M_{\text {colored }}$ for $f_{H}=1 \mathrm{TeV}$. Dashed curves correspond to different choices of $\left(M_{1}, m_{4}, \theta_{R}\right)$, as listed in the legend. For comparison, the black solid line corresponds to $\mathrm{MCHM}_{5+1}$ without a tadpole (i.e., with $\beta=\beta_{\mathrm{SM}}$ generated by large $q_{L}$ compositeness, determining $\left.\theta_{R}\right)$.

radiative Higgs potential is given in appendix A. In the limit of a fully composite $t_{R}$, $\sin \theta_{R}=1$ and

$$
\delta \alpha=-\frac{3 y_{t}^{2}}{16 \pi^{2}} \frac{M_{4}^{2}}{f_{H}^{2}}\left(1+\log \left(\frac{\mu^{2}}{M_{4}^{2}}\right)\right)
$$

The one-loop quadratic divergences are cut-off, but a residual logarithmic scale-dependence remains associated with the scale $\mu$ of the next set of top partner resonances [21]. For concreteness, we set $\log \frac{\mu}{M_{4}}=\log \frac{\mu}{M_{1}}=\log 3$, but our results are not particularly sensitive to this choice.

Figure 2 shows the tuning as a function of the lightest top partner mass for several different sets of parameters $\left(M_{1}, m_{4}, \theta_{R}\right)$, with $\sin \theta_{L}$ determined from eq. (2.11). For comparison, we also show the tuning for the $\mathrm{MCHM}_{5+1}$ model without a tadpole in which the minimal top sector generates $\beta=\beta_{\mathrm{SM}}$ radiatively to give $m_{h}=125 \mathrm{GeV}$. In this case, achieving sufficiently large $\beta=\beta_{\mathrm{SM}}$ requires an increase in $q_{L}$ compositeness, such that the explicit global symmetry breaking increases and the tuning becomes more severe. ${ }^{1}$ Induced EWSB removes the restriction of obtaining $\beta=\beta_{\mathrm{SM}}$ from the top sector, allowing the lower tunings present in the alternative more natural top sectors to be achieved. Since $|\delta \alpha|>|\delta \beta|$ in the $\mathrm{MCHM}_{5+1}$ model, the tuning still tends to be somewhat worse than the minimal tuning, $\Delta \gtrsim \frac{f_{H}^{2}}{2 v_{H}^{2}}$. But, the tadpole mechanism in the $\mathrm{SO}(5) / \mathrm{SO}(4)$ model allows $m_{h}=125 \mathrm{GeV}$ to be obtained with the minimal representations of the fermion partners $\left(\mathrm{MCHM}_{5+1}\right)$ and a tuning of $\sim 5 \%$, a significant improvement over the $\sim 0.5 \%$ tuning exhibited by an $\mathrm{MCHM}_{5+1}$ model in which $\beta=\beta_{\mathrm{SM}}$ is radiatively generated.

\footnotetext{
${ }^{1}$ Similar to raising $m_{h}$ via large $A$-terms in the MSSM - the increased explicit symmetry breaking enhances the quartic, but also results in more tuning.
} 
Essentially because the top partners cannot be made lighter than $\sim 2 f_{H}$, the rough bound eq. (2.8) is not reached by the $\mathrm{MCHM}_{5+1}$ model even with the additional of a tadpole sector. So, the overall naturalness is not necessarily improved relative to every MCHM model with a radiatively generated potential. For example, a larger top partner representation as found in the $\mathrm{MCHM}_{14+1}$ model can nearly saturate $\Delta \sim f_{H}^{2} / 2 v_{H}^{2}$ and obtain $\sim 5 \%$ tuning for $f_{H} \sim m_{*} \sim \mathrm{TeV}[21]$. So tadpole-induced EWSB resuscitates some $\mathrm{SO}(5) / \mathrm{SO}(4)$ composite Higgs models with minimal top partner representations, but does not necessarily improve upon minimally-tuned $\mathrm{SO}(5) / \mathrm{SO}(4)$ models with extended representations. $\mathrm{By}$ comparison, in the Twin Higgs models we will study in section 3.2, the neutral top partners can be sufficiently light to realize the lower limit of eq. (2.8), such that the induced structure offers a substantial improvement in naturalness over any radiative model.

\subsection{Dynamical auxiliary sectors}

So far, we have considered a tadpole that arises due to an unspecified auxiliary sector exhibiting an $\mathrm{SU}(2)_{L}$-breaking vev $f_{\Sigma}$. However, the dynamics of the auxiliary sector are also relevant. For instance, the auxiliary sector experiences back-reaction from the non-zero Higgs vev, and it is important to ensure that this does not destabilize the auxiliary sector or lead to hidden tuning. Meanwhile, any explicit $G$-breaking present in the auxiliary sector may be communicated to the Higgs sector.

The presence of an additional sector containing an electroweak doublet also leads to modifications of Higgs properties and novel states that may be produced at colliders. Notably, a second doublet gives rise to additional charged and pseudoscalar Higgses, $H^{ \pm}$ and $A$ respectively, similar to those of a fermiophobic/type-I two Higgs doublet model (in which only a single doublet couples to fermions). Thus, the largely SM-like nature of the Higgs and the non-observation of BSM states at the LHC constrains the dynamics of the auxiliary sector. Overall, the auxiliary sector must exhibit certain properties in order to remain stable against back-reaction, to stay consistent with experimental measurements, and to preserve the improved naturalness of the model.

One important question is whether the auxiliary sector is weakly-coupled Sigma model or a strongly-coupled theory (e.g. bosonic technicolor) - i.e., is $\Sigma$ elementary or composite? Several considerations disfavor a weakly-coupled auxiliary sector. First, experimental constraints on Higgs couplings require $f_{\Sigma} \ll v_{H}$. So, for $v_{H} \ll f_{H}$, eq. (2.7) implies

$$
\frac{\mu^{2}}{f_{\Sigma}^{2}}=\frac{m_{h}^{2} v}{f_{\Sigma}^{3}} \approx 10\left(\frac{70 \mathrm{GeV}}{f_{\Sigma}}\right)^{3}
$$

This is very similar to the size $\mu^{2} \sim 4 \pi f_{\Sigma}^{2}$ suggested by naïve dimensional analysis for a strongly-coupled auxiliary sector with an $\mathcal{O}(1)$ weak coupling to the Higgs sector. Second, large couplings help stabilize $f_{\Sigma} \ll v_{H}$ against large back-reaction when the Higgs field acquires its vev. Finally, large couplings raise the mass scale of the resonances associated with the auxiliary sector, explaining their non-observation thus far at the LHC.

A second issue is that the $\Sigma$ sector need not respect the approximate global symmetry $G$ - in fact, explicit $G$-breaking in the $\Sigma$ sector can avoid additional light modes and may reduce its susceptibility to back-reaction. The details of the $\Sigma$ sector determine how this 
explicit breaking is communicated to the Higgs sector. The low-energy form of the coupling $\mu^{2} H \cdot \Sigma$ is a soft breaking of $G$ in the Higgs sector, and so the contributions to the pNGB potential will be under control even for a strongly-coupled auxiliary sector that generates important higher-order terms. However, for some UV completions of the $\Sigma$ sector there can be further UV-sensitive contributions to the pNGB potential.

In this section, we shall explore the structure of a linearly-realized auxiliary sector. As the issues discussed above likely imply strong coupling, this model is more useful for developing intuition than it is realistic. However, considering this model in the strong selfcoupling limit allows us to move beyond the frozen tadpole approximation and investigate the back-reaction, tuning and impact of $\Sigma$-sector $G$-breaking described above, as well as the form of the tadpoles generated. Because the $\Sigma$ sector must be near strong coupling and its interactions with the Higgs sector can be a strong perturbation, there may be important higher-order effects neglected in this description. But, while these operators may have interesting implications, we find that the qualitative features of the model remain unchanged. We refer the interested reader to appendix B.1, in which we discuss stronglycoupled auxiliary sectors more explicitly, focusing on the additional higher-order operators between the Higgs and $\Sigma$ sectors we expect in this scenario.

An effective theory analysis has previously been carried out in the context of a simplified model of induced EWSB with a single Higgs doublet coupled to a linearly-realized $\Sigma$ doublet in [41]. They confirmed that it was possible to achieve a stable vacuum with $f_{\Sigma}<v_{H}$ and, as the tadpole limit is approached, tuning does indeed become small. Here, we extend this analysis to the case of the MCHM. Starting with the $\mathrm{SO}(5) / \mathrm{SO}(4)$ case, we take the $\Sigma$ sector to be a simple linear model,

$$
V_{\Sigma}=-\Lambda_{\Sigma}^{2}|\Sigma|^{2}+\delta_{\Sigma}|\Sigma|^{4}
$$

which only realizes the custodial $\mathrm{SO}(4)$ symmetry. In the absence of a coupling to the Higgs, $\mathrm{SO}(4)$ is spontaneously broken at scale $f_{\Sigma}^{2}=\frac{\Lambda_{\Sigma}^{2}}{\delta_{\Sigma}}$. The Higgs and auxiliary sectors are linked by a $B \mu$-type term,

$$
V \supset-\mu^{2} \Sigma^{\dagger} H+\text { h.c. }
$$

producing the necessary EWSB tadpole. In addition, this term explicitly breaks $\mathrm{SO}(5)_{H} \times$ $\mathrm{SO}(4)_{\Sigma} \rightarrow \mathrm{SO}(4)$, both giving mass to the extra Higgs states $m_{A}^{2} \simeq m_{H^{ \pm}}^{2} \propto \mu^{2}$ and inducing $\mathrm{SU}(2)_{L}$-alignment between $\langle H\rangle$ and $\langle\Sigma\rangle$.

For simplicity, in this linearly-realized example we disregard quartic couplings between $H$ and $\Sigma$, e.g., $\left(H \cdot \Sigma^{\dagger}\right)^{2}$. These marginal operators can be made irrelevant in a UV completion of the theory, and the effects of radiatively generating such operators in the IR theory are captured by the strong-coupling analysis of appendix B.1.

To estimate the impact of back-reaction on the auxiliary sector, we focus on the neutral CP-even states, expanding about the unperturbed $\Sigma$ vacuum $|\Sigma|=\frac{f_{\Sigma}+\sigma}{\sqrt{2}}$ and treating the Higgs pNGB as a background field. This gives a quadratic potential

$$
V_{\Sigma}=\Lambda_{\Sigma}^{2} \sigma^{2}-\mu^{2}\left(f_{H} s_{h}\right) \sigma .
$$


The effective tadpole for $\sigma$ shifts the $\Sigma$-sector EWSB vev

$$
\langle\sigma\rangle \simeq \frac{\mu^{2} f_{H} \sin \left(\frac{v_{H}}{f_{H}}\right)}{2 \Lambda_{\Sigma}^{2}} .
$$

The auxiliary sector minimization condition combined with eq. (2.13) implies

$$
\frac{\langle\sigma\rangle}{f_{\Sigma}} \simeq \frac{m_{h}^{2} v_{H}^{2}}{\delta_{\Sigma} f_{\Sigma}^{4}} \simeq 0.5\left(\frac{4 \pi^{2}}{\delta_{\Sigma}}\right)\left(\frac{70 \mathrm{GeV}}{f_{\Sigma}}\right)^{4} .
$$

So, the EWSB vev in the $\Sigma$ sector does receive a correction due to back-reaction from the Higgs vev, but this effect is suppressed in the strong coupling regime when $\delta_{\Sigma}$ is large. ${ }^{2}$ In particular, that the shift in $\langle\Sigma\rangle$ is relatively small in this regime indicates that backreaction does not result in additional tuning, and the theory remains under qualitative control for $f_{\Sigma} \gtrsim 50 \mathrm{GeV}$.

Meanwhile, the Higgs experiences explicit $\mathrm{SO}(5)$-breaking in addition to the tadpole through its interactions with $\sigma$. In this simplified picture, this breaking can be viewed as communicated via mixing of the $\mathrm{CP}$-even states, which induces higher-order operators in the pNGB potential. It is useful to define $\epsilon=\frac{\mu^{2}}{2 \Lambda_{\Sigma}^{2}}$ to parameterize the mixing angle of the Higgs $\mathrm{pNGB}$ and $\sigma$,

$$
\epsilon \simeq 0.14\left(\frac{4 \pi^{2}}{\delta_{\Sigma}}\right)\left(\frac{70 \mathrm{GeV}}{f_{\Sigma}}\right)^{4}
$$

Again, these effects are suppressed in the large-coupling limit. Integrating out $\sigma$ gives rise to new terms in the pNGB potential, including

$$
V_{h} \supset-\epsilon^{2} \Lambda_{\Sigma}^{2} f_{H}^{2} s_{h}^{2} \simeq-\frac{\mu^{4}}{4 \Lambda_{\Sigma}^{2} f_{H}^{2}} f_{H}^{4} s_{h}^{2}
$$

corresponding to a contribution to $\alpha$

$$
\left|\frac{(\delta \alpha)_{\Sigma}}{\alpha}\right| \simeq 0.5\left(\frac{4 \pi^{2}}{\delta_{\Sigma}}\right)\left(\frac{70 \mathrm{GeV}}{f_{\Sigma}}\right)^{4} .
$$

In the strong-coupling limit, this effect is of similar size to the experimentally-required value of $\alpha$, and therefore does not induce additional tuning. Higher-order terms are suppressed by powers of mixing between the Higgs and $\Sigma$ sector, but can be relevant for the phenomenology of the extra Higgs states, as mentioned in appendix B.1.

This analysis indicates that the dynamics of the auxiliary sector do not disrupt the leading-order description of a Higgs pNGB with positive mass term $(\alpha>0)$ and EWSB induced by a tadpole as in section 2.1, particularly in the strong-coupling limit suggested by experimental constraints. Back-reaction and explicit $\mathrm{SO}(5)$-breaking lead to at most $\mathcal{O}(1)$ shifts to $\left(f_{\Sigma}, \alpha\right)$, and so for strong-coupling induce no additional tuning in either sector.

\footnotetext{
${ }^{2}$ For our chosen normalization of the quartic, nonperturbative self-coupling corresponds to $\delta_{\Sigma} \rightarrow 4 \pi^{2}$.
} 


\subsection{UV considerations}

Finally, we highlight some of the additional important issues that should be addressed by UV completions attempting to explain the origin of the pNGB Higgs and strongly coupled auxiliary sectors.

As stressed throughout, the mechanism of induced EWSB requires $\alpha>0$. This does not present a particular challenge in the MCHM, where there are positive contributions from the gauge sector quadratically sensitive to the vector resonance masses, giving $\alpha_{0} \sim \frac{g_{2}^{2}}{16 \pi^{2}} M_{\rho}^{2}[5,54]$. This can be sufficiently large when the vector resonances are heavy compared to the top partners, $M_{\rho} \gtrsim 3 M_{*}$.

Depending on the structure of the $\Sigma$ sector, there can also be UV contributions to the Higgs potential $\propto \mu^{2}$, which may need to be suppressed to avoid tuning. For example, $\Sigma$ can emerge from an asymptotically free technicolor-like sector that is weakly coupled at the scale $\Lambda_{H}$ with $\mathcal{O}_{\Sigma}$ formed from a bilinear of technifermions, $\mathcal{O}_{\Sigma}=\Sigma^{I}+\ldots \simeq \frac{1}{f_{\Sigma} \Lambda_{\Sigma}} \psi_{\Sigma} \bar{\psi}_{\Sigma}$. Contributions to the potential for $H$ are cut off at $\Lambda_{H}^{2}$ and give a leading one-loop UV contribution

$$
V_{\mu^{2}, \mathrm{UV}} \sim \frac{\Lambda_{H}^{2}}{16 \pi^{2}}\left(\frac{\mu_{I j}^{2}}{\Lambda_{\Sigma} f_{\Sigma}} H^{I}\right)^{2} \simeq \Lambda_{\Sigma}^{2} f_{H}^{2}\left(\frac{\mu^{2} f_{H}}{\Lambda_{\Sigma}^{2} f_{\Sigma}}\right)^{2} s_{h}^{2}
$$

This exceeds the IR-generated quadratic term by a factor $\propto \frac{f_{H}^{2}}{f_{\Sigma}^{2}}$, so could dominate over the radiative top sector tuning if unsuppressed. The UV $s_{h}^{4}$ term is of comparable size to the IR-generated term, and higher-order UV terms are subdominant. This sensitivity can be avoided in a theory where the scaling dimension is $\left[\mathcal{O}_{\Sigma}\right] \lesssim 2$, in which case the contributions are effectively cutoff at $\Lambda_{\Sigma}$ instead of $\Lambda_{H}$. For example, this can occur if the completion of the $\Sigma$ sector is instead a conformal-technicolor theory [55] with large anomalous dimension for the fermion bilinears $\left(\left[\mathcal{O}_{\Sigma}\right] \leq 2\right.$ is also trivially satisfied in the scalar linear $\Sigma$ model).

A UV completion should also address the potentially dissatisfying coincidence of scales, $f_{\Sigma} \lesssim m_{h} \sim v$. In the context of SUSY, for EWSB induced by a strongly-coupled $\Sigma$ sector, ref. [41] suggested that the auxiliary sector could be near a strongly-coupled superconformal fixed point in the UV. SUSY breaking triggers confinement at a scale close to that of the scalar soft masses. One could imagine a similar mechanism here, namely that confinement in a nearly-conformal auxiliary sector is triggered by breaking of the approximate global symmetry at $\Lambda_{H}$ (though, admittedly, there are more known examples of superconformal theories), for example by the coupling of $\mathrm{SO}(5)$ singlet operators in the $H$ sector to an $\mathrm{SO}(4)$ singlet operator in the auxiliary sector. Regardless of the solution, it must avoid introducing a hierarchy problem in the $\Sigma$ sector, which would of course spoil the improved naturalness exhibited by these models.

\section{Twin Higgs model}

Twin Higgs models extend the coset and elementary states of a pNGB Higgs model beyond the MCHM to preserve a spontaneously broken $\mathbb{Z}_{2}$ mirror symmetry, giving new mirror top and gauge partners at the scale $v_{B} \sim f_{H}$. The restored $\mathbb{Z}_{2}$ symmetry is sufficient to cut 
off the quadratic sensitivity of the pNGB potential at the scale $f_{H}$ instead of the scale of the colored top partners, which are somewhat heavier. The extra protection from radiative contributions to the potential combined with the tadpole structure will allows these models to evade the 'irreducible' $\frac{f_{H}^{2}}{2 v_{H}^{2}}$ tuning giving, for example, a technically natural model of EWSB with a global symmetry-breaking scale $f_{H} \sim 1 \mathrm{TeV}$ and colored top partners at $2 \mathrm{TeV}$.

The original twin Higgs model $[6,7]$ consisted of an $\mathrm{SU}(4)$-invariant potential

$$
V=-M^{2}\left(\left|H_{A}\right|^{2}+\left|H_{B}\right|^{2}\right)+\lambda\left(\left|H_{A}\right|^{2}+\left|H_{B}\right|^{2}\right)^{2},
$$

where $H_{A, B}$ are doublets of weakly-gauged $\mathrm{SU}(2)_{A, B} \subset \mathrm{SU}(4)$, with a small $\mathrm{SU}(4)$-violating but $\mathbb{Z}_{2}$-preserving quartic

$$
V \supset \delta\left(\left|H_{A}\right|^{4}+\left|H_{B}\right|^{4}\right) .
$$

The $\mathbb{Z}_{2}$ parity exchanges $A$ and $B$. In strongly-coupled realizations a larger $\mathrm{SO}(8)$ symmetry should be considered $[7,50,51,56] .{ }^{3}$ When the approximate $\mathrm{SU}(4)$ is spontaneously broken by a large vev $f_{H} \gg v_{H}$, there is an uneaten pNGB that is associated with the Higgs, which develops a potential proportional to explicit SU(4) breaking. Parameterizing

$$
\left|H_{A}\right|^{2}=\frac{f_{H}^{2}}{2} \sin ^{2}\left(\frac{h}{f_{H}}\right), \quad\left|H_{B}\right|^{2}=\frac{f_{H}^{2}}{2} \cos ^{2}\left(\frac{h}{f_{H}}\right),
$$

one finds a potential for the light Higgs mode of the form of eq. (2.1) with $\beta=-\alpha=\frac{\delta}{2}$. The $\mathbb{Z}_{2}$ symmetry ensures that quadratically-divergent radiative contributions take the form $\Lambda^{2}\left(\left|H_{A}\right|^{2}+\left|H_{B}\right|^{2}\right)$, which is independent of the light Higgs field.

For $\delta>0$, as for the IR contribution of a $\mathbb{Z}_{2}$-preserving top sector, the unbroken parity would enforce $\left|H_{A}\right|^{2}=\left|H_{B}\right|^{2}=\frac{f_{H}^{2}}{4}$. In this case, achieving $\left|H_{A}\right|^{2}=\frac{v_{H}^{2}}{2} \ll \frac{f_{H}^{2}}{2}$ (associating the $\mathrm{SM}$ weak gauge group with $\left.\mathrm{SU}(2)_{A}\right)$ requires explicit $\mathbb{Z}_{2}$ breaking. In the original model, this was accomplished by a soft $\mathbb{Z}_{2}$-breaking mass term

$$
\Delta V=\Delta m^{2}\left(\left|H_{A}\right|^{2}-\left|H_{B}\right|^{2}\right),
$$

giving an additional contribution to $\alpha \sim \frac{\Delta m^{2}}{f_{H}^{2}}$. This contribution can be fine-tuned against the above contribution $\delta \alpha=-\frac{\delta}{2}$ to get the correct vev, but also results in the tuning described for the conventional models. If the top sector generates the observed value of $\delta=2 \beta_{\mathrm{SM}}$, these models exhibit a minimal tuning $\Delta \geq \frac{f_{H}^{2}}{2 v_{H}^{2}}$.

In the remainder of this section, we extend the detailed analysis of the MCHM model with a tadpole of section 2 to the case of the Twin Higgs.

\subsection{Twin Higgs with a tadpole}

Introducing an auxiliary EWSB sector can readily remove the $\frac{f_{H}^{2}}{2 v_{H}^{2}}$ tuning of the Twin Higgs model. We assume that, prior to EWSB, the SU(4)-breaking vev is stabilized at $\left|H_{A}\right|^{2}=0,\left|H_{B}\right|^{2}=\frac{f_{H}^{2}}{2}$. This can be achieved in the limit of unbroken $\mathbb{Z}_{2}$, for instance

\footnotetext{
${ }^{3}$ For a review of some of the flavor phenomenology of Composite Twin Higgs, see [57].
} 
if $\delta<0$, or due to the presence of a large $\Delta m^{2}>\delta f_{H}^{2}-$ we will return to the possible origin of the various terms in section 3.4. The auxiliary sector is assumed to preserve the $\mathbb{Z}_{2}$ symmetry and the tadpole terms are

$$
\Delta V=-\mu^{2}\left(\Sigma_{A} \cdot H_{A}+\Sigma_{B} \cdot H_{B}\right)+\text { h.c. }
$$

Below the scale $f_{H}$, in the frozen tadpole approximation $\left\langle\Sigma_{A}\right\rangle=\left\langle\Sigma_{B}\right\rangle=f_{\Sigma}$, the Higgs potential takes the form

$$
\begin{aligned}
V(h)= & -\mu^{2} f_{\Sigma} f_{H}\left(\sin \left(\frac{h}{f_{H}}\right)+\cos \left(\frac{h}{f_{H}}\right)\right) \\
& +\alpha f_{H}^{4} \sin ^{2}\left(\frac{h}{f_{H}}\right)-\beta f_{H}^{4} \sin ^{4}\left(\frac{h}{f_{H}}\right)
\end{aligned}
$$

such that the Higgs vev is determined by

$$
-2 \beta \sin ^{3}\left(\frac{\langle h\rangle}{f_{H}}\right)+\alpha \sin \left(\frac{\langle h\rangle}{f_{H}}\right)+\frac{\mu^{2} f_{\Sigma}}{f_{H}^{3}} \tan \left(\frac{\langle h\rangle}{f_{H}}\right)=\frac{\mu^{2} f_{\Sigma}}{f_{H}^{3}} .
$$

For $\beta \sim \alpha$ and $f_{H} \gg v_{H}, \tan \left(\frac{v_{H}}{f_{H}}\right) \simeq \sin \left(\frac{v_{H}}{f_{H}}\right)$ and the cubic term can be approximately neglected. So, the correct vev is simply achieved by the tadpole

$$
\mu^{2} f_{\Sigma} \simeq \frac{\alpha f_{H}^{3} \sin \left(\frac{\langle h\rangle}{f_{H}}\right)}{1-\sin \left(\frac{\langle h\rangle}{f_{H}}\right)}=\alpha f_{H}^{2} v_{H}\left(1+\mathcal{O}\left(\frac{\langle h\rangle}{f_{H}}\right)\right) .
$$

Just as before, the tadpole allows the vev to be continuously perturbed away from the $v_{H}=0$ vacuum, giving a hierarchy $v_{H} \ll f_{H}$ without any tuning.

It is interesting to note that, while the enlarged structure of the Twin Higgs due to the $\mathbb{Z}_{2}$ symmetry permits multiple possibilities for the unperturbed vacuum (i.e., with $\mu^{2}=0$ ), this reduced tuning is unique to the model perturbing around $\left(\left|H_{A}\right|,\left|H_{B}\right|\right)=\left(0, \frac{f_{H}}{\sqrt{2}}\right)$ with $\mathbb{Z}_{2}$-symmetric tadpoles. In principal, spontaneous or explicit $\mathbb{Z}_{2}$ breaking in the $\Sigma$ sector could give a tadpole only in the $A$-sector, $\left(\left\langle\Sigma_{A}\right\rangle,\langle\Sigma\rangle\right)=\left(\frac{f_{\Sigma}}{\sqrt{2}}, 0\right)$. But, in this case, the vacuum with $v_{H} \ll f_{H}$ reached by perturbing about $\left(\left|H_{A}\right|,\left|H_{B}\right|\right)=\left(0, \frac{f_{H}}{\sqrt{2}}\right)$ is always unstable to a global vacuum at $v_{H}=f_{H}$ reached from the unperturbed $\left(\left|H_{A}\right|,\left|H_{B}\right|\right)=$ $\left(\frac{f_{H}}{\sqrt{2}}, 0\right)$ vacuum. Moreoever, this is true even in the presence of higher-order terms that may induce a misalignment in the $\mathrm{SU}(2)$ orientation of the $H_{A, B}$ and $\Sigma_{A, B}$ vevs. Alternatively, ref. [58] considered a similar model with the unperturbed vacuum instead at $\left(\left|H_{A}\right|,\left|H_{B}\right|\right)=$ $\left(\frac{f_{H}}{2}, \frac{f_{H}}{2}\right)$ and a spontaneous $\mathbb{Z}_{2}$ breaking in the tadpole sector, $\left(\left\langle\Sigma_{A}\right\rangle,\left\langle\Sigma_{B}\right\rangle\right)=\left(0, f_{\Sigma}\right)$. The tadpole helps favor $\left\langle\left|H_{A}\right|\right\rangle\left\langle\left\langle\left|H_{B}\right|\right\rangle\right.$, but obtaining a hierarchy $v_{H} \ll f_{H}$ is still a large perturbation away from the unperturbed vacuum, requiring a tuning of the tadpole against the parameters of the Higgs sector.

Thus $v_{H} \ll f_{H}$ can be obtained naturally in the Twin Higgs model with a tadpole $\gamma=\mu^{2} f_{\Sigma} / f_{H}^{3}$ that is protected against the radiative contributions generating the mass term $\alpha$. Provided radiative contributions to $\alpha$ are sufficiently small, $\delta \alpha \sim \frac{m_{h}^{2}}{f_{H}^{2}}$, such a model can be considerably less tuned than the original Twin Higgs model. We discuss the improved naturalness of induced EWSB for concrete examples of Twin top sectors in the next section. 


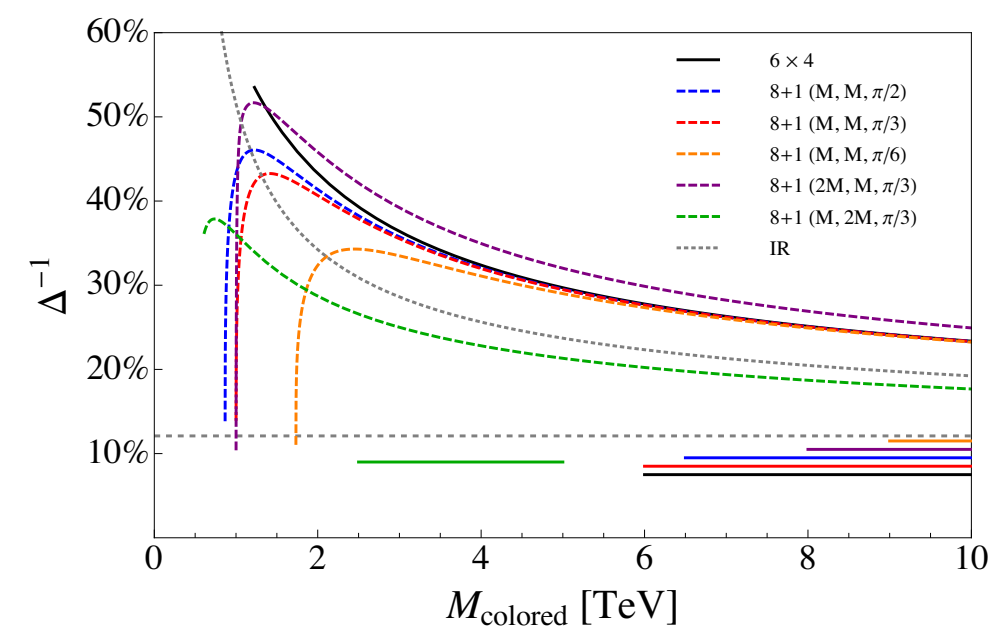

Figure 3. Top sector radiative tuning in a Twin Higgs model with a tadpole as a function of the lightest colored top partner mass $M_{\text {colored }}$ for $f_{H}=1 \mathrm{TeV}$. Dotted gray is the estimated tuning from the pure $t_{B}$ contribution of eq. (3.9). Solid black is the $6 \times 4$ model, while dashed, colored curves correspond to the $8+1$ model with $\left(M_{1}, m_{7}, \theta_{R}\right)$ as listed. For comparison, the horizontal dotted gray line corresponds to the minimal tuning $\frac{2 v_{H}^{2}}{f_{H}^{2}} \simeq 10 \%$ of the radiative quartic potential, with horizontal lines indicate the top partner mass range which can radiatively generate $\beta=\beta_{\mathrm{SM}}$ (saturating this tuning) within theoretical uncertainty.

\subsection{Radiative tuning}

The quadratic sensitivity of $\alpha$ to the top sector in Twin Higgs models is cut off by the twin top at $m_{t_{B}} \simeq \frac{y_{t} f_{H}}{\sqrt{2}}$, but a logarithmic sensitivity remains to the scale $M_{T}$ of new colored top partners that restore the full global symmetry in the top sector,

$$
\delta \alpha \simeq-\frac{3 y_{t}^{4}}{32 \pi^{2}} \log \frac{M_{T}^{2}}{m_{t_{B}}^{2}} .
$$

We will study two concrete models of Twin top sectors to determine the degree to which light colored top partners can lower the radiative tuning of the tadpole potential with respect to the minimal $\frac{f_{H}^{2}}{2 v_{H}^{2}}$ tuning of the purely radiative potential. Current direct experimental bounds require only $M_{T} \gtrsim 700 \mathrm{GeV}$ and will not significantly constrain the naturalness of these models. However, realizing the observed top Yukawa coupling and including threshold contributions to eq. (3.9) again gives a lower bound on the tuning.

Again motivated by bounds on Higgs properties, we will fix $f_{H}=1 \mathrm{TeV}\left(\frac{f_{H}^{2}}{v_{H}^{2}} \simeq 16\right)$ and $\left\langle\Sigma_{A}\right\rangle=\left\langle\Sigma_{B}\right\rangle=f_{\Sigma}=60 \mathrm{GeV}$ as a benchmark in this section. The results are summarized in figure 3 , which compares the tuning in several models to the logarithmic estimate eq. (3.9). Unsurprisingly, we find that the minimal tuning occurs for top partners with masses roughly just above the smallest possible value required to realize the top Yukawa, $M_{T} \simeq \sqrt{2} f_{H} \sim$ $m_{t_{B}}$. For these values, induced EWSB can reduce tuning by a factor of $\sim 5$ relative to the minimal $\frac{2 v_{H}^{2}}{f_{H}^{2}} \sim 10 \%$ tuning of the radiative quartic potential. 
$6 \times 4$ top sector. Ref. [6] proposed completing the top sector by extending $\left(Q_{A}, Q_{B}\right)$ into a $Q=(6, \overline{4})$ of $\mathrm{SU}(6) \times \mathrm{SU}(4)$, with $\mathrm{SU}(3)_{c, A} \times \mathrm{SU}(3)_{c, B} \subset \mathrm{SU}(6) . Q$ contains new top partners $\left(\tilde{q}_{A}, \tilde{q}_{B}\right)$ required to restore the global symmetry in the $\left(3_{A}, 2_{B}\right)$ and $\left(3_{B}, 2_{A}\right)$ representations. The Yukawa coupling $y H Q U$ respects the $\mathrm{SU}(4)$ symmetry and the exotic mixed states can be lifted by soft $\mathrm{SU}(4)$-breaking vector-like masses $M\left(\tilde{q}_{A} \tilde{q}_{A}^{c}+\tilde{q}_{B} \tilde{q}_{B}^{c}\right)$. We will refer to this as the ' $6 \times 4$ ' model.

To leading order in $\frac{v_{H}}{f_{H}}$, the colored top partner mass is $M_{T_{A}}^{2}=M^{2}+\frac{y^{2} f_{H}^{2}}{2}$ while the uncolored mirror top and top partner have masses $m_{t_{B}}^{2}=\frac{y^{2} f_{H}^{2}}{2}$ and $M_{T_{B}}^{2}=M^{2}$ respectively. ${ }^{4}$ The coupling $y$ is related to the top Yukawa coupling as $y^{2}=y_{t}^{2}\left(1-\frac{y_{t}^{2} f_{H}^{2}}{2 M^{2}}\right)^{-1}$, such that there is a minimal value for the colored top partner mass $M_{T_{A}} \geq \sqrt{2} y_{t} f_{H}$. The radiative contribution to $\alpha$ is

$$
\delta \alpha=\frac{3}{16 \pi^{2}} \frac{y^{2} M^{2} / f_{H}^{2}}{M^{2}-\frac{y^{2} f_{H}^{2}}{2}}\left(M^{2} \log \frac{M_{T_{A}}^{2}}{M_{T_{B}}^{2}}-\frac{y^{2} f_{H}^{2}}{2} \log \frac{M_{T_{A}}^{2}}{m_{t_{B}}^{2}}\right) .
$$

We evaluate eq. (3.10) using the SM $\overline{\mathrm{MS}}$ value of the top mass at $\mu=m_{t_{B}} \simeq 700 \mathrm{GeV}$.

Figure 3 shows the radiative tuning due to this top sector. Also shown in figure 3 is the approximate range of colored top partner mass $M \sim 10 \mathrm{TeV}$ that gives $\beta=\beta_{\mathrm{SM}}$ and would saturate the $2 v_{H}^{2} / f_{H}^{2}$ tuning in the absence of the tadpole (we estimate the theoretical uncertainty by varying the top Yukawa coupling between its $\overline{\mathrm{MS}}$ values at $\mu=m_{t}$ and $\left.\mu=m_{t_{B}}\right)$. For $M<3 f_{H}$, the tuning becomes considerably less than the 'irreducible' tuning exhibited when $\beta=\beta_{\mathrm{SM}}$. At $M \simeq f_{H}$, the coupling $y$ becomes large and the tuning begins to worsen. For $M \gg f_{H}, \delta \alpha$ matches the expected logarithmic behavior eq. (3.9). The minimally-tuned tadpole potential can permit significantly lower colored top partner masses, and correspondingly substantially reduced tuning.

$8+1$ top sector. Refs. [50, 51] studied pNGB Twin Higgs models based on an $\mathrm{SO}(8) / \mathrm{SO}(7)$ coset with a partially composite top sector, similar to those studied in the MCHM [5, 20, 21] and above. In particular we focus on the model studied in ref. [51] with $q_{L}$ embedded in an $8=7+1, t_{R}$ in a singlet, and composite top partners $\psi_{A}=\left(\psi_{7, A}^{i}, \psi_{1, A}\right)$ and $\psi_{B}^{i}=\left(\psi_{7, B}^{i}, \psi_{1, B}\right)$ in a $\left(3_{A}, 8\right)$ and $\left(3_{B}, 8\right)$ respectively. This is the Twin analog of the $\mathrm{MCHM}_{5+1}$ model.

In a two-site model for this composite-sector, the radiative contributions to the Higgs potential can be calculated directly and parameterized in terms of two top partner mass scales $m_{1}$ and $m_{7}$ and the mixing angles $\sin \theta_{L, R}$ of the top quark with the composites $\psi_{A}, \psi_{A}^{c}$. To leading order in $\frac{v_{H}}{f_{H}}$, the colored $\left(3_{A}, 2_{A}\right)$ top partners are at masses $m_{7}$ and $M_{7}=m_{7} / \cos \theta_{L}$, and the $\left(3_{A}, 2_{B}\right)$ top partners are at a mass $M_{1}=m_{1} / \cos \theta_{R}$. The Yukawa coupling is

$$
y_{t}=\frac{m_{7}}{f_{H}} \sin \theta_{L} \sin \theta_{R}
$$

to leading order, which requires $m_{7} \gtrsim f_{H}$.

The full definition of the two-site model and expressions for the radiative corrections are described in appendix A following ref. [51]. In the Twin model the contributions to

\footnotetext{
${ }^{4}$ Our normalization of $f_{H}$ differs by a factor of $\sqrt{2}$ from ref. [6].
} 
$\alpha$ are only logarithmically sensitive to the colored top partner masses, and therefore the residual scale dependence found in the two-site $5+1$ model is absent.

Figure 3 shows the tuning of the tadpole potential for the $8+1$ model in terms of the parameters $\left(M_{1}, m_{7}, \theta_{R}\right)$, with $\sin \theta_{L}$ fixed by the top Yukawa, eq. (3.11). Again, we highlight the top partner masses that would give $\beta=\beta_{\mathrm{SM}}$ and so saturate the $\frac{f_{H}^{2}}{2 v_{H}^{2}}$ tuning (i.e., in the absence of the tadpole). We observe an improvement in tuning by a factor of $\sim 5$ is possible with the tadpole. The improvement is substantial over most of the parameter space with $m_{7} \lesssim 3 f_{H}$, but the tuning begins to worsen as the physical mass $M_{7}$ gets large at the lower range of $m_{7}$. Note that the improvement in tuning by a factor of $\sim 10$ compared to the $\mathrm{SO}(5) / \mathrm{SO}(4) 5+1$ model studied in section 2.2 can be understood as a result of uncolored top partners cutting off the quadratic sensitivity at a substantially lower scale than that at which colored top partners can appear.

\subsection{Dynamical twin auxiliary sector}

The dynamics of the auxiliary sector coupling to a Twin Higgs model are similar to the $\mathrm{SO}(5) / \mathrm{SO}(4)$ scenario described in 2.3 with one important difference, namely that the $\mathbb{Z}_{2}$ symmetry requires that both of the scales in the $H$ sector, including $v_{B} \sim f_{H} \gg v_{H}$, couple to the auxiliary sector. This causes a larger perturbation in the $\Sigma$ sector, although such perturbations can still be sufficiently small to avoid tuning or destabilization of the auxiliary sector. Moreover, the additional interactions between sectors may offer some intriguing opportunities, including generation of the required $\alpha>0$, dynamical generation of the scales $f_{\Sigma} \ll v_{H} \ll f_{H}$ and complete $\mathrm{SU}(2)_{B} \times \mathrm{U}(1)_{B}$-breaking. Again, we will illustrate this behavior with a simple linear $\Sigma$ model. The strongly coupled model is more carefully analyzed in appendix B.2.

Extending the potential eq. (2.14) to the Twin Higgs case, we consider a "Twin Sister" model ${ }^{5}$ with

$$
V_{\Sigma} \supset-\Lambda_{\Sigma}^{2}\left(\left|\Sigma_{A}\right|^{2}+\left|\Sigma_{B}\right|^{2}\right)+\lambda_{\Sigma}\left(\left|\Sigma_{A}\right|^{2}+\left|\Sigma_{B}\right|^{2}\right)^{2}+\delta_{\Sigma}\left(\left|\Sigma_{A}\right|^{4}+\left|\Sigma_{B}\right|^{4}\right)
$$

The Higgs sector is of the same form as given in eqs. (3.1) and (3.2) with $\delta \ll \lambda$ giving the approximate $\mathrm{SU}(4)_{H}$ symmetry. For simplicity, we take $\delta_{\Sigma} \gg \lambda_{\Sigma}$ and treat the $\lambda_{\Sigma}$ term coupling the $\Sigma_{A}$ and $\Sigma_{B}$ sectors as a perturbation. The unperturbed vev is then

$$
\left\langle\Sigma_{A}\right\rangle^{2}=\left\langle\Sigma_{B}\right\rangle^{2}=\frac{f_{\Sigma_{A, B}}^{2}}{2}=\frac{\Lambda_{\Sigma}^{2}}{\delta_{\Sigma}}
$$

and $\mathrm{SU}(4)_{\Sigma}$ is explictly broken to $\mathrm{SU}(2)_{\Sigma_{A}} \times \mathrm{SU}(2)_{\Sigma_{B}}$. The $H$ and $\Sigma$ sectors are again linked by a $B \mu$-type term,

$$
V \supset-\mu^{2}\left(\Sigma_{A}^{\dagger} H_{A}+\Sigma_{B}^{\dagger} H_{B}+\text { h.c. }\right)
$$

which is an explicit soft breaking of the $\mathrm{SU}(2)_{\Sigma_{A}} \times \mathrm{SU}(2)_{\Sigma_{B}} \times \mathrm{SU}(4)_{H}$ global symmetry to the gauge and discrete symmetry $\mathrm{SU}(2)_{A} \times \mathrm{SU}(2)_{B} \times \mathbb{Z}_{2}$.

\footnotetext{
${ }^{5}$ A twinning of the Sister Higgs [59].
} 
Following the same strategy of integrating out the $\Sigma$ sector, we have the leading quadratic terms

$$
V_{\Sigma}=\Lambda_{\Sigma}^{2}\left(\sigma_{A}^{2}+\sigma_{B}^{2}\right)-\mu^{2} f_{H}\left(s_{h} \sigma_{A}+c_{h} \sigma_{B}\right)+\lambda_{\Sigma} f_{\Sigma}^{2} \sigma_{A} \sigma_{B}
$$

where we have elided terms proportional to $\lambda_{\Sigma}$ that do not couple the $\Sigma_{A}$ and $\Sigma_{B}$ sectors. The $B$-sector vev is shifted by

$$
\begin{aligned}
\frac{\left\langle\sigma_{B}\right\rangle}{f_{\Sigma}} & =\epsilon \frac{f_{H} \cos \left(\frac{v}{f_{H}}\right)}{f_{\Sigma}} \\
& \simeq 2\left(\frac{4 \pi^{2}}{\delta_{\Sigma}}\right)\left(\frac{70 \mathrm{GeV}}{f_{\Sigma}}\right)^{4}\left(\frac{1 \mathrm{TeV}}{f_{H}}\right) .
\end{aligned}
$$

As anticipated, if there is a hierarchy $f_{H} \gg v_{H}$, this can be an $\mathcal{O}(1)$ perturbation even as $\delta_{\Sigma}$ approaches strong coupling.

Likewise, there can be significant contributions to the Higgs potential. The leading contribution present in the $\mathrm{SO}(5) / \mathrm{SO}(4)$ case, eq. (2.20), is cancelled because of the $\mathbb{Z}_{2}$ Twin protection. The $\mathbb{Z}_{2}$ breaking shift in $\langle\sigma\rangle$ is captured by the $\sigma^{3}, \sigma^{4}$ terms, which give

$$
V_{h} \supset \delta_{\Sigma} f_{\Sigma} \epsilon^{3}\left(s_{h}^{3}+c_{h}^{3}\right)+\frac{\delta_{\Sigma}}{4} \epsilon^{4}\left(s_{h}^{4}+c_{h}^{4}\right)
$$

potentially producing contributions $\left|\frac{(\delta \alpha)_{\Sigma}}{\alpha}\right| \simeq \mathcal{O}$ (few). In particular, as $\left\langle\Sigma_{B}\right\rangle$ is unconstrained by experiment, it can be somewhat larger than $\left\langle\Sigma_{A}\right\rangle$, such that contributions to the Higgs mass can be somewhat enhanced. This indicates the possibility that Higgs couplings to the auxiliary sector may be the source of the required $\alpha_{0}>0$.

The back-reaction and mixing contributions remain comparable to the required $f_{\Sigma_{A, B}}$ and $\alpha$. So, while they make a complete analysis of the potential somewhat more complicated, they do not induce significant additional tuning. We have confirmed this with a full numerical study of the potential in Mathematica; for instance, with $f_{H} \simeq 1 \mathrm{TeV}$, $f_{\Sigma_{A}}=70 \mathrm{GeV}, f_{\Sigma_{B}}=150 \mathrm{GeV}$ and strong couplings $\sim 4 \pi^{2}$ in the $\Sigma$ sector, it is possible to achieve the observed $m_{h}$ and EWSB with $\Delta \lesssim 4$. Overall, our results are consistent with those of [41]; it is possible to achieve $\mathcal{O}(1)$ tuning and a stable vacuum with $\left\langle\Sigma_{A}\right\rangle \lesssim\left\langle\Sigma_{B}\right\rangle \ll v_{H} \ll f_{H}$. A realistic auxiliary sector likely exhibits approximately $\mathbb{Z}_{2^{-}}$ symmetric vevs, a large explicit breaking of the global symmetry (i.e., $\delta_{\Sigma} \gtrsim \lambda_{\Sigma}$ ), and strong coupling.

So far, we have ignored the role of $\lambda_{\Sigma}$. Treating $\lambda_{\Sigma}$ as a perturbation, a shift in $\left\langle\Sigma_{A}\right\rangle$ is also generated at leading order as a result of $\left\langle\sigma_{B}\right\rangle,\left\langle\sigma_{A}\right\rangle=\frac{\lambda_{\Sigma}}{2 \delta_{\Sigma}}\left\langle\sigma_{B}\right\rangle$. Clearly, for a more generic potential with $\lambda_{\Sigma} \sim \delta_{\Sigma}$, both $\left\langle\Sigma_{A}\right\rangle$ and $\left\langle\Sigma_{B}\right\rangle$ can experience large perturbations due to the $f_{H}$ tadpole. This raises the interesting possibility that the hierarchy and coincidence of scales is generated by a "waterfall" of induced breakings originating from $f_{H}$. For instance, in the limit that $\Lambda_{\Sigma}=0$, the scale of the $\Sigma$ sector is set completely by the large $B$-sector tadpole from $f_{H}$. While $\Lambda_{\Sigma}=0$ is unnatural in the linear sigma model, this serves as a useful toy model for a strongly-coupled model where the scale of a conformal $\Sigma$ sector may be triggered by the coupling to the $H$ sector, as we will discuss shortly. Then 
$\delta_{\Sigma}^{3 / 2} f_{\Sigma_{B}}^{3} \simeq \mu^{2} f_{H}$, and a term $\lambda_{\Sigma}<0$ can trigger the breaking in the $A$-sector. This waterfall of breaking then feeds back into the $H_{A}$ sector through the EWSB-inducing tadpole.

As for the $\mathrm{MCHM}_{5+1}$, this linear sigma model nicely illustrates all the key features of the Twin Higgs tadpole mechanism, in particular that the model remains valid even beyond the frozen tadpole approximation employed above. Likewise, the Twin Model remains under control and has the same qualitative behavior as the linear sigma model even in the strongly coupled regime.

\subsection{UV issues}

Finally, in the Twin case, there are some further interesting aspects of UV completions beyond those addressed for the MCHM in section 2.4.

First, unlike the MCHM, for the Twin Higgs the origin of $\alpha_{0}>0$ cannot be contributions from the gauge sector, as the $\mathbb{Z}_{2}$ symmetry removes the quadratic sensitivity. Fortunately, the higher-order contributions from coupling the pNGB Higgs to the twin auxiliary sector are larger in this case, so can provide a sufficient enough source of $\alpha_{0}>0$, especially when the negative top sector contributions are near the natural size $\alpha_{\text {obs }}$. Alternatively, additional soft $\mathbb{Z}_{2}$ breakings can generate $\alpha_{0}>0$.

Another question is whether $\Sigma_{A}$ and $\Sigma_{B}$ are part of a single strongly-interacting gauge sector $\mathcal{G}$ or two independent strongly-interacting sectors $\mathcal{G}_{A}$ and $\mathcal{G}_{B}$ related by the $\mathbb{Z}_{2}$. The former naturally admits the appealing "waterfall" of induced breakings described in the preceding subsection. For a single strongly-coupled sector, we expect sizable couplings between $\Sigma_{A}$ and $\Sigma_{B}$, corresponding to $\lambda_{\Sigma} \sim \delta_{\Sigma}$ in the linear model. The condensation in the Higgs sector at $\Lambda_{H}$ generates a scale in the $B$ auxiliary sector, triggering its condensation. For example, the auxiliary sector could be a conformal technicolor-like sector near a strongly-coupled fixed point at $\Lambda_{H}$, with some techniquarks $\mathcal{O}_{\Sigma} \sim \psi_{\Sigma} \bar{\psi}_{\Sigma}$ acquiring $\mathrm{SU}(2)_{A}$-preserving masses proportional to $f_{H}$. This triggers a chiral symmetry-breaking phase for both the $A$ and $B$ sectors, which in turn generates the tadpole for $H_{A}$, inducing EWSB. In this scenario, the scale in the $A$ auxiliary sector is directly related to the scale in the $B$ auxiliary sector so we expect $\left\langle\Sigma_{B}\right\rangle \sim\left\langle\Sigma_{A}\right\rangle$ in the absence of tuning. The scales of the Higgs and $\Sigma_{A}$ sector are therefore directly connected as $\Lambda_{\Sigma_{A}}^{3} \sim \mu^{2} f_{H}$, and the viable parameter space $\left\langle\Sigma_{A}\right\rangle \sim 50-70 \mathrm{GeV}$ requires $f_{H} \sim \mathrm{TeV}$. Alternatively, if $\mathcal{G}_{\mathcal{A}}$ and $\mathcal{G}_{\mathcal{B}}$ are independent, the Twin sector can induce $\left\langle\Sigma_{B}\right\rangle \gg\left\langle\Sigma_{A}\right\rangle$ which can increase the size of the extra contributions to the Higgs potential.

\section{Experimental constraints}

Induced EWSB is subject to both indirect constraints from measurements of Higgs properties and electroweak precision tests, and direct constraints from searches for additional states associated with the auxiliary sector. These constraints have been extensively studied in [42], with emphasis on phenomenological models and applications to supersymmetry. Notably, there exists a tension between electroweak precision tests and direct searches for vector resonances, which favor larger values of $f_{\Sigma}$, and Higgs measurements (both of Higgs properties and searches for extended Higgs sector states), which favor smaller values of 
$f_{\Sigma}$. Here, we summarize these results, and highlight some of the main differences in the MCHM or Twin scenario.

The presence of an additional source of EWSB modifies Higgs couplings to SM states. If the auxiliary sector is strongly-coupled, this results in a universal enhancement of Higgs couplings to fermions and a suppression of couplings to gauge bosons, parameterized by the ratios

$$
\begin{gathered}
\kappa_{f} \equiv \frac{g_{h f \bar{f}}}{g_{h f \bar{f})}^{(\mathrm{SM})}}=\frac{1}{\sqrt{1-\frac{f_{\Sigma}^{2}}{v^{2}}}}, \\
\kappa_{V} \equiv \frac{g_{h V V}}{g_{h V V}^{(\mathrm{SM})}}=\sqrt{1-\frac{f_{\Sigma}^{2}}{v^{2}}} .
\end{gathered}
$$

The allowed values of $f_{\Sigma}$ are thus constrained by the combined ATLAS and CMS Higgs measurements [17-19] — for a strongly-coupled auxiliary sector, $f_{\Sigma} \lesssim 0.3 v$ [42]. Motivated by the discussion of section 2.3 , we focus on strongly-coupled auxiliary sectors here. However we do note that, if the auxiliary sector is at least somewhat weakly-coupled, the constraints vary due to the mixing between the Higgs and the radial mode of the auxiliary sector. This mode couples to gauge bosons but not to fermions, so mixing partially restores the depletion of $\kappa_{V}$ while also reducing the enhancement of $\kappa_{f}$.

In pNGB Higgs models, there is additional universal suppression of Higgs couplings due to $\frac{v_{H}^{2}}{f_{H}^{2}}$ corrections,

$$
\kappa_{h}^{(\mathrm{pNGB})} \simeq \sqrt{1-\frac{v_{H}^{2}}{f_{H}^{2}}} .
$$

While this counteracts the enhancement of Higgs coupling to fermions, it also further suppresses coupling to vector bosons. Since current measurements favor a slight enhancement $\kappa_{V}=1.05>1$, constraints on $f_{\Sigma}$ can be somewhat more stringent for smaller values of $f_{H}$ than in the usual induced EWSB scenario described above.

For Twin Higgs models, there is further additional suppression of Higgs couplings to visible SM states due to decays to Twin sector states [60]. For instance, supposing the couplings to SM and Twin $b$ quarks respect the $\mathbb{Z}_{2}$, the Higgs is expected to decay to Twin $b$ 's with width $\Gamma_{h \rightarrow b^{\prime} \bar{b}^{\prime}} \simeq \frac{v_{H}^{2}}{f_{H}^{2}} \Gamma_{h \rightarrow b \bar{b}}$, leading to a suppression factor

$$
\kappa_{h}^{(\mathrm{TH})} \simeq \frac{1}{\sqrt{1+\frac{v_{H}^{2}}{f_{H}^{2}} \mathrm{Br}^{(\mathrm{SM})}(h \rightarrow b \bar{b})}}
$$

where $\mathrm{Br}^{(\mathrm{SM})}(h \rightarrow b \bar{b})=0.577$ for $m_{h}=125 \mathrm{GeV}$. However, depending on the exact details of the quark couplings, this decay may be suppressed and a variety of Higgs decays to Twin sector states, including displaced decays, may be possible (see, e.g., [61-63]).

In figure 4 , we plot the $\left(\kappa_{V}, \kappa_{f}\right)$ that can occur in induced EWSB models with a pNGB Higgs and a strongly-coupled auxiliary sector, as well as the combined ATLAS and CMS measurements [17]. We consider both a general MCHM model (i.e., with additional suppression $\kappa_{h}^{(\mathrm{pNGB})}$ relative to eqs. (4.1) and (4.2) only), as well as a TH model with 


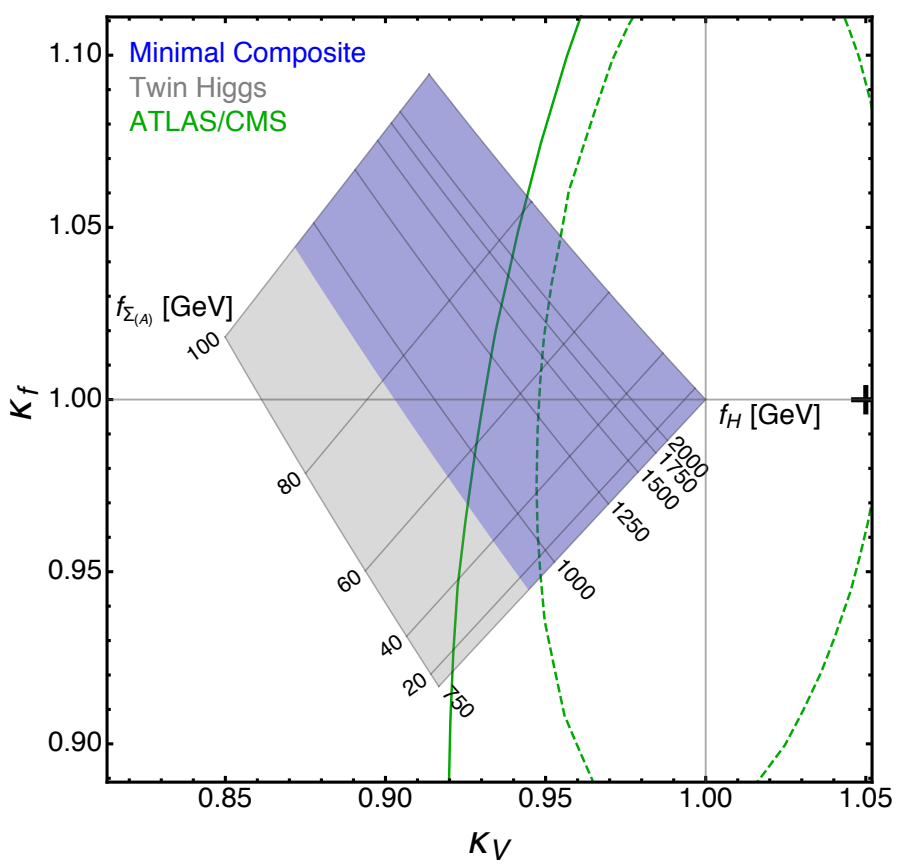

Figure 4. Values of $\left(\kappa_{V}, \kappa_{f}\right)$ in Minimal Composite (blue) and Twin (gray) models for $0 \leq f_{\Sigma} \leq$ $100 \mathrm{GeV}$ and $750 \mathrm{GeV} \leq f_{H}$. Contours correspond to values of $f_{\Sigma}$ and $f_{H}$ in a Twin Higgs model with unsuppressed decays to Twin $b$ 's. Solid elliptical contour corresponds to the combination of current ATLAS and CMS measurements, with central value $\left(\kappa_{V}, \kappa_{f}\right)=(1.05,1)$ [17]. Dashed contour corresponds to projections from [42] assuming central value $\left(\kappa_{V}, \kappa_{f}\right)=(1,1)$.

unsuppressed decays to Twin $b$ 's (with additional suppression $\kappa_{h}^{(\mathrm{pNGB})} \kappa_{h}^{(\mathrm{TH})}$ ). We also show projected limits from [42] assuming $\sqrt{s}=14 \mathrm{TeV}, \mathcal{L}=300 \mathrm{fb}^{-1}$ and central value $\left(\kappa_{V}, \kappa_{f}\right)=(1,1)$. Note that although we plot over an extended range of $f_{\Sigma}$ for illustration, as discussed in section 2.3 the theory is outside of good theoretical control when $f_{\Sigma} \lesssim$ $50 \mathrm{GeV}$.

There are also constraints from direct searches for states associated with the auxiliary sector, which generally require these states to be at least somewhat heavy. First, there are the additional Higgs sector states due to the presence of a second electroweak doublet $\Sigma_{(A)}$. Direct searches for heavy Higgs bosons constrain $m_{A}$, with the dominant constraint in much of the parameter space coming from the CMS search for $A \rightarrow Z h \rightarrow \ell^{+} \ell^{-} b \bar{b}$ [64], which requires $m_{A} \gtrsim 460 \mathrm{GeV}$ [42]. In these models, the extra Higgs states have masses related to the size of the $H \cdot \Sigma$ terms connecting the two sectors, since these are the only terms breaking the independent $\mathrm{SU}(2)_{\Sigma}$ and $\mathrm{SU}(2)_{H}$ symmetries. When only the tadpole bilinear term is present, the extra Higgs states have masses [42]

$$
m_{A}^{2}=m_{H^{ \pm}}^{2}=m_{h}^{2} \frac{v^{2}}{f_{\Sigma}^{2}}\left(1+\mathcal{O}\left(v_{H}^{2} / f_{H}^{2}\right)\right) .
$$

For concreteness, we present our results evaluating the $v_{H}^{2} / f_{H}^{2}$ corrections with $\beta=0$ for the MCHM and $\beta=\alpha$ for the Twin Higgs. For strongly-coupled models, an even more important effect comes from higher-order $H \cdot \Sigma$ terms which can yield $\mathcal{O}(1)$ corrections to 


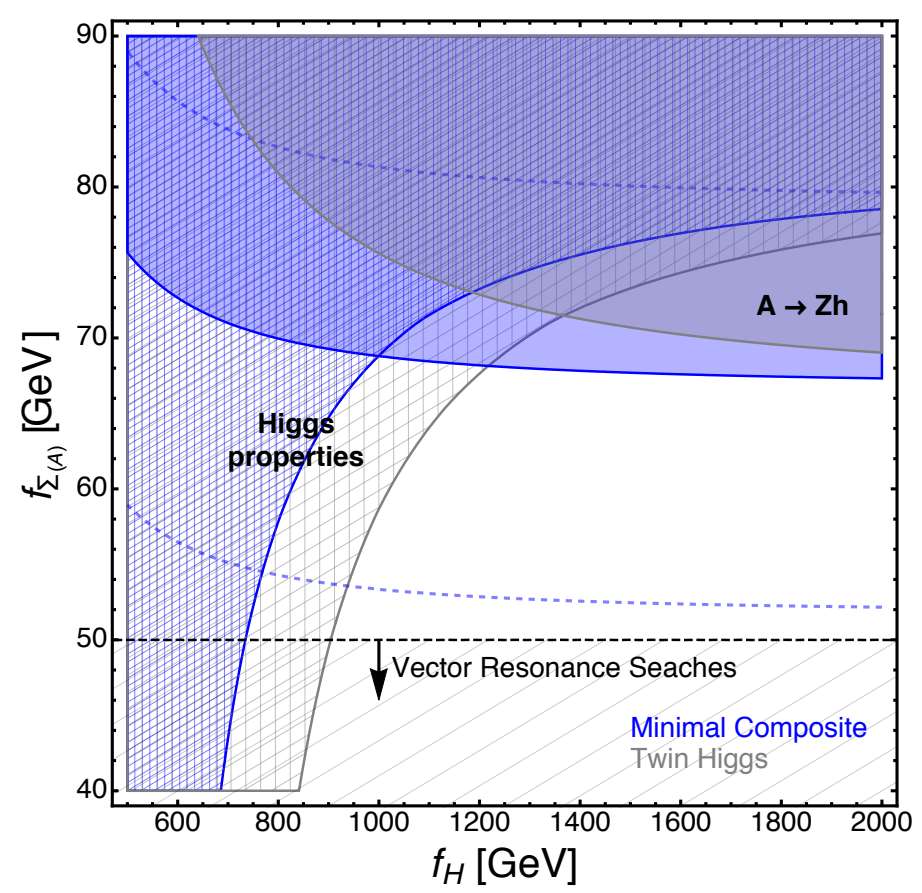

Figure 5. Regions of $\left(f_{H}, f_{\Sigma}\right)$ excluded by Higgs coupling measurements (hatched) and direct $A \rightarrow Z h$ searches (solid) for Minimal Composite (blue) and Twin (gray) Higgs models. Solid regions correspond to $m_{A}$ with $\beta=0$ for MCHM and $\beta=\alpha$ for Twin Higgs, see text for details. Dashed blue contours represent the effect of rescaling $m_{A}^{2}$ by 0.6 (lower) or 1.4 (upper) and thus represent the theoretical uncertainty on the solid blue line. The dashed black line denotes approximate lower bound $f_{\Sigma} \gtrsim 50 \mathrm{GeV}$ from vector resonance searches.

eq. (4.5) (as mentioned in appendix B). This gives a 'theoretical uncertainty' in the relation between $\left(f_{H}, f_{\Sigma}\right)$ and the mass of Higgs resonances in the auxiliary sector. In figure 5 , we show approximate constraints from $A \rightarrow Z h$ and Higgs property measurements in the $\left(f_{H}, f_{\Sigma}\right)$ plane. To capture the theoretical uncertainty, we show the impact of rescaling $m_{A}^{2} \rightarrow(0.6,1.4) m_{A}^{2}$ for the MCHM. An uncertainty band of similar proportion also applies for the Twin Higgs. Note that although $m_{H^{ \pm}} \simeq m_{A}$, searches for charged Higgses are currently less constraining [42].

A second set of constraints comes from vector resonances. If the auxiliary sector is indeed strongly-coupled, we expect vector resonances with masses $m_{\rho} \sim 4 \pi f_{\Sigma}$ associated with the strong dynamics [65]. These "technirhos" are constrained both by direct searches (notably, $\rho^{ \pm} \rightarrow W^{ \pm} Z$ [66]) and by electroweak precision measurements [32]. The exact constraints depend on the properties of the technirhos, which depend on the details of the unknown strong dynamics. However, for lighter technirhos (such as those predicted by a QCD-like auxiliary sector), these can be the dominant constraints, eliminating the majority of the allowed parameter space [42]. Thus, for a truly strongly-coupled auxiliary sector, the strong dynamics must be such that the vector resonances are at least somewhat heavy. For instance, the (non-excluded) strongly-coupled benchmarks considered in [42] would require $f_{\Sigma} \gtrsim 50-55 \mathrm{GeV}$. Meanwhile, perturbativity generally places an upper bound on $m_{\rho}$. 
Finally, for pNGB Higgs models, top partner searches are of course also relevant. There are a variety of searches focusing on a minimal charge-2/3 top partner decaying via $T \rightarrow b W, t Z, t h$, which currently require $m_{T} \gtrsim 700 \mathrm{GeV}$ [12-14]. A top partner of this variety is expected to be somewhat light as it is responsible for cutting off quadratic divergences due to the SM top quark. However, in 'maximally natural' models, the full global symmetry is likely restored not too far above $m_{T}$ (see section 2.2). As a result, searches for other states implied by the global symmetry, such as heavy charge- $1 / 3 \mathrm{~B}$ quarks $[67,68]$ or exotic charge-5/3 quarks $[67,69]$ (present in complete multiplets of custodial $\mathrm{SO}(4)$ ) may also be relevant [70, 71]. In particular, for Twin Higgs models, the lightest top partner responsible for regulating the quadratic divergences is uncolored, leading to weak constraints from the LHC. But natural models likely exhibit colored top partners not too much heavier than the uncolored twin top (as in section 3.2), which may be probed up to $m_{*} \sim 2.5 \mathrm{TeV}$ at the LHC [72].

\section{Conclusion}

Tadpole-induced electroweak symmetry breaking gives an alternative structure for the lowenergy potential of a pNGB Higgs model. This structure allows the desired EWSB pattern with $m_{h}=125 \mathrm{GeV}$ and $v_{H} \ll f_{H}$ to be achieved in Composite Higgs models that could not otherwise realize a large enough quartic term $\beta$ without excessive tuning. Unlike other tree-level modifications to the pNGB Higgs potential, which focus on increasing the quartic term $\beta$ (e.g., Little Higgs), the tadpole structure simply makes $\beta$ irrelevant in the limit $v_{H} \ll f_{H}$.

In $\mathrm{SO}(5) / \mathrm{SO}(4)$ minimal composite Higgs models (MCHM), this mechanism makes viable the minimal representations of the $3 \mathrm{rd}$ generation partners (as in $\mathrm{MCHM}_{5+1}$ ). The resulting tuning is comparable to a purely radiative potential generated by 3rd generation partners in larger representations. In the case of the Twin Higgs, the radiative contributions from the minimal representations of the top sector can be made substantially smaller, and the mechanism of induced EWSB allows the tuning to be reduced by a factor of $\sim 5$ compared to the 'irreducible' $\frac{f_{H}^{2}}{2 v_{H}^{2}}$ tuning of a purely radiative potential. This allows a fully natural pNGB potential with $f_{H} \simeq 1 \mathrm{TeV}$ and colored top partners at $\sim 2 \mathrm{TeV}$. The tadpole mechanism in the Twin Higgs model also has the advantage of incorporating spontaneous $\mathbb{Z}_{2}$ breaking and full breaking of the mirror $\mathrm{U}(1)_{\mathrm{EM}, \mathrm{B}}$.

While these pNGB Higgs models share many features in common with supersymmetric models of induced EWSB [27, 28, 36-42], there are interesting differences. First, in SUSY models, both the $H$ and $\Sigma$ sectors inherit their scale from an external SUSY breaking sector, while in the composite pNGB case the scale $f_{H}$ can directly trigger $f_{\Sigma}$. Second, although in both cases the striking phenomenology is in the Higgs sector, Higgs compositeness generates additional deviations in Higgs properties not present in SUSY. If the fermionic top partners of the pNGB Higgs model are within reach, their signatures also differ substantially from the signatures of the scalar stops in SUSY models.

In the most appealing version of the model, the scale $f_{H}$ of global symmetry breaking triggers a waterfall of breaking where $f_{H}$ dynamically induces the smaller $f_{\Sigma}$ which in 
turn induces $v_{H}$, naturally connecting the scales $f_{\Sigma} \ll v_{H} \ll f_{H}$. In this scenario, the compositeness scale must be $f_{H} \sim \mathrm{TeV}$. Meanwhile, the combination of Higgs property measurements and searches for the new auxiliary sector states set both upper and lower bounds on the scale $f_{\Sigma}$, and it is non-trivial that there is consistent parameter space for this model with new $\mathrm{TeV}$-scale physics.

In tadpole-induced pNGB Higgs models, a wealth of interesting phenomenology from both the $\Sigma$ sector and Higgs compositeness may be within reach of the LHC. The plethora of signals could include modifications of Higgs properties due to both compositeness and the auxiliary EWSB component, extra charged and pseudoscalar Higgs states, auxiliary sector vector resonances lighter than $1 \mathrm{TeV}$, and colored composite top partners at $\gtrsim \mathrm{TeV}$. In the case of the Twin Higgs, further consequences of the mirror sector, including invisible and/or exotic Higgs decays, may be observable. It has not escaped our attention that the auxiliary sector generically contains composite singlet pseudoscalars at the scale $\Lambda_{\Sigma} \sim$ $4 \pi f_{\Sigma} \sim 750 \mathrm{GeV}$ with large branching ratios to diphotons [73-79], which may be able to explain recent hints for a resonance at LHC13 [80, 81]. In particular, small mixings between the auxiliary sector and singlet pseudoscalars in the composite Higgs sector [82-85] can lead to an appreciable gluon fusion production cross section even if the auxiliary sector contains no colored states.

Not only can tadpole-induced models feature a pNGB potential with a fully natural scale for EWSB, but in fact searches at LHC13 and future colliders will likely be able to probe the entire remaining range of viable models independent of any naturalness arguments.

\section{Acknowledgments}

We thank Nathaniel Craig for useful discussions at various stages of this work and also wish to congratulate him for his productivity. We also thank Zackaria Chacko, Roberto Contino, Juliano Panico, Ennio Salvioni, Yuhsin Tsai, and Andrea Tesi. We thank Alex Kagan and Adam Martin for friendly coordination regarding their upcoming related paper [86]. Fermilab is operated by Fermi Research Alliance, LLC under Contract No. DE-AC0207CH11359 with the United States Department of Energy.

\section{A Expressions for two-site models}

\section{A.1 $5+1$}

The Lagrangian for the two-site model defining the mass mixings and Yukawa couplings of the $5+1$ top sector is $\mathcal{L}=-m_{1}\left(\psi_{1} \psi_{1}^{c}\right)-m_{4}\left(\psi_{4}^{i} \psi_{4}^{c i}\right)-m_{R}\left(\psi_{1} t_{R}\right)-\frac{y_{L} f_{H}}{\sqrt{2}}\left(t_{L}\left(\psi_{4}^{c(2)}+\cos \frac{h}{f_{H}} \psi_{4}^{c(4)}\right)+\sin \frac{h}{f_{H}} t_{L} \psi_{1}^{c}\right)+$ h.c.

The breaking of the global symmetry due to the top sector mixings is completely parameterized by $y_{L}$. The mixing angles are $\sin ^{2} \theta_{R}=\frac{m_{R}^{2}}{m_{R}^{2}+m_{1}^{2}}$ and $\sin ^{2} \theta_{L}=\frac{y_{L}^{2} f_{H}^{2}}{y_{L}^{2} f_{H}^{2}+m_{4}^{2}}$, and the top partners mixing with the elementary sector obtain masses $M_{1}^{2}=m_{1}^{2}+m_{R}^{2}$ 
and $M_{4}^{2}=m_{4}^{2}+y_{L}^{2} f_{H}^{2}$. The Top Yukawa coupling is given to leading order in $\sin \frac{h}{f_{H}}$ as $y_{t}=\frac{m_{4}}{f} \sin \theta_{R} \sin \theta_{L}$.

The mass matrix can be diagonalized perturbatively in $\sin \frac{h}{f_{H}}$, giving a ColemanWeinberg contribution to the effective potential,

$$
\Delta \alpha=-\frac{3 y_{L}^{2}}{16 \pi^{2} f^{2}}\left(m_{4}^{2}\left(1+\log \frac{\mu^{2}}{M_{4}^{2}}\right)-m_{1}^{2}\left(1+\log \frac{\mu^{2}}{M_{1}^{2}}\right)+\frac{m_{1}^{2} y_{L}^{2} f_{H}^{2}}{M_{4}^{2}-M_{1}^{2}} \log \frac{M_{4}^{2}}{M_{1}^{2}}\right) .
$$

$\Delta \beta$ is obtained in the same fashion.

\section{A. $28+1$}

The Lagrangian for the two-site model defining the mass mixings and Yukawa couplings of the $8+1$ top sector generalizes the $5+1$ model to the twin case by extending the coset to $\mathrm{SO}(8) / \mathrm{SO}(7)$ and including B-sector elementary and composite tops,

$$
\begin{aligned}
\mathcal{L}= & -m_{1}\left(\psi_{1, A} \psi_{1, A}^{c}+\psi_{1, B} \psi_{1, B}^{c}\right)-m_{7}\left(\psi_{7, A}^{i} \psi_{7, A}^{c}{ }^{i}+\psi_{7, B}^{i} \psi_{7, B}^{c}{ }^{i}\right)-m_{R}\left(\psi_{1, A} t_{R, A}+\psi_{1, B} t_{R, B}\right) \\
& -\frac{y_{L} f_{H}}{\sqrt{2}}\left(t_{L, A}\left(\psi_{7, A}^{c}{ }^{(2)}+\cos \frac{h}{f_{H}} \psi_{7, A}^{c}{ }^{(4)}\right)+\sin \frac{h}{f_{H}} t_{L, A} \psi_{1, A}^{c}\right) \\
& -\frac{y_{L} f_{H}}{\sqrt{2}}\left(t_{L, B}\left(\psi_{7, B}^{c}{ }^{(6)}+\cos \frac{h}{f_{H}} \psi_{1, B}^{c}\right)+\sin \frac{h}{f_{H}} t_{L, B} \psi_{7, B}^{c}{ }^{(4)}\right)+\text { h.c. }
\end{aligned}
$$

It is simplest to proceed directly to the radiative potential following ref. [51]. We obtain $\alpha$ by expanding to order $\sin ^{2} \frac{h}{f}$ (ref. [51] makes a similar expansion in $y^{2}$ instead of $\left.\sin ^{2} \frac{h}{f}\right)$,

$$
\begin{aligned}
\Delta \alpha=-\int & \frac{d^{4} p}{(2 \pi)^{4}} \frac{y_{L}^{4}\left(m_{1}^{2} p^{2}+m_{7}^{2}\left(m_{R}^{2}-p^{2}\right)\right)^{2}}{p^{2}\left(-m_{1}^{2}-m_{R}^{2}+p^{2}\right)\left(-f_{H}^{2} y_{L}^{2}-m_{7}^{2}+p^{2}\right)} \\
& \times \frac{1}{\left(m_{1}^{2} p^{2}\left(f_{H}^{2} y_{L}^{2}+2 m_{7}^{2}-2 p^{2}\right)+\left(m_{R}^{2}-p^{2}\right)\left(m_{7}^{2}\left(2 p^{2}-f_{H}^{2} y_{L}^{2}\right)+2 f_{H}^{2} p^{2} y_{L}^{2}-2 p^{4}\right)\right)} .
\end{aligned}
$$

$\Delta \beta$ is obtained in the same fashion.

\section{B Strongly-coupled auxiliary sectors}

\section{B.1 Composite Higgs}

Here, we consider more explicitly the case that $H$ and $\Sigma$ emerge from independent stronglycoupled sectors with compositeness scales $\Lambda_{\Sigma}<\Lambda_{H}$ using the appropriate non-linearly realization. The global symmetries of the two sectors are $\mathrm{SO}(5)_{H}$ and $\mathrm{SO}(4)_{\Sigma}$. At scales above $\Lambda_{H}$, the two sectors are weakly coupled by an operator explicitly breaking $\mathrm{SO}(5)_{H} \times$ $\mathrm{SO}(4)_{\Sigma} \rightarrow \mathrm{SO}(4)$

$$
\mathcal{L} \supset \mathcal{O}_{\mu^{2}}=\mu_{I j}^{2} \mathcal{O}_{H}^{I} \mathcal{O}_{\Sigma}^{j}
$$


The spurion $\mu_{I j}^{2}$ parameterizes the breaking,

$$
\mu_{I j}^{2}=\mu^{2}\left(\begin{array}{llll}
1 & 0 & 0 & 0 \\
0 & 1 & 0 & 0 \\
0 & 0 & 1 & 0 \\
0 & 0 & 0 & 1 \\
0 & 0 & 0 & 0
\end{array}\right) .
$$

We normalize these operators so that, in terms of the low-energy goldstone fields, $\mathcal{O}_{H}^{I}=$ $H^{I}(1+\ldots)$ and $\mathcal{O}_{\Sigma}^{j}=\Sigma^{j}(1+\ldots)$. The neglected terms are higher derivative in the goldstone fields. A convenient realization of the pNGB manifold is given by

$$
\begin{aligned}
& H_{I}=\frac{f_{H}}{\sqrt{2}} e^{i \Pi_{h}^{a} T_{a} / v_{H}}\left(\begin{array}{c}
0 \\
0 \\
0 \\
s_{h} \\
0
\end{array}\right)+\frac{f_{H}}{\sqrt{2}}\left(\begin{array}{c}
0 \\
0 \\
0 \\
0 \\
c_{h}
\end{array}\right), \\
& \Sigma_{I}=\frac{f_{\Sigma}}{\sqrt{2}} e^{i \Pi_{\Sigma}^{a} T_{a} / f_{\Sigma}}\left(\begin{array}{l}
0 \\
0 \\
0 \\
1
\end{array}\right)
\end{aligned}
$$

The fields $\Pi_{h}^{a}$ and $\Pi_{\Sigma}^{a}$ correspond to the pNGBs of the broken $\mathrm{SU}(2)_{H}$ and $\mathrm{SU}(2)_{\Sigma}$, with a linear combination

$$
\Pi_{G}^{a}=\frac{v_{H}}{v} \Pi_{h}^{a}+\frac{f_{\Sigma}}{v} \Pi_{h}^{a}
$$

absorbed by the gauge bosons and the remaining

$$
\Pi_{A}^{a}=\frac{f_{\Sigma}}{v} \Pi_{h}^{a}-\frac{v_{H}}{v} \Pi_{\Sigma}^{a}
$$

obtaining a mass from the explicit breaking.

When $\mathcal{O}_{\mu^{2}}$ is a sufficiently weak perturbation on both the $\Sigma$ and $H$ sectors, the leading effect in the IR at scales below $\Lambda_{H}$ is to generate the tadpole term $\mu^{2} H \cdot \Sigma$ in the pNGB potential. In the parameter space of interest $\mathcal{O}_{\mu^{2}}$ will always be a weak perturbation on the fundamental $H$ sector at $\Lambda_{H}$, but it may not be a weak perturbation on the $\Sigma$ sector; by NDA [87-89] $\mathcal{O}_{\mu^{2}}$ can be a strong perturbation on the $\Sigma$ sector if $\mu^{2} v_{H} \gtrsim \Lambda_{\Sigma}^{2} f_{\Sigma}$, as for the linear sigma model above.

The effects on the pNGB Higgs potential are determined by treating $H$ as a background field and integrating out the $\Sigma$ sector at $\Lambda_{\Sigma}$ to obtain the full Goldstone potential,

$$
V_{\mu^{2}}\left(h, \Pi_{A}\right) \equiv \hat{V}\left(\frac{\Sigma^{j}}{f_{\Sigma}}, \frac{\mu_{I j}^{2} H^{I}}{\Lambda_{\Sigma}^{2} f_{\Sigma}}\right) \Lambda_{\Sigma}^{2} f_{\Sigma}^{2}
$$

with $\hat{V}$ a function with $\mathcal{O}(1)$ coefficients. Terms of the form $\mu_{I j}^{2} \Sigma^{j} H^{I}$ generate a potential for both $h$ and $\Pi_{A}$, while the invariant $\mu_{K j}^{2} \mu_{I j}^{2} H^{K} H^{I}$ generates a potential only for $h$. For 
the Higgs potential, we obtain simply

$$
V_{\mu^{2}}(h)=\Lambda_{\Sigma}^{2} f_{\Sigma}^{2} \hat{V}\left(\frac{\mu^{2} f_{H}}{\Lambda_{\Sigma}^{2} f_{\Sigma}} s_{h}\right) .
$$

This term fully describes the IR contributions from the $\Sigma$ sector, and connects the size of the tadpole to the higher-order terms. For instance, these terms will generate a contribution to $\alpha$,

$$
\delta \alpha \simeq \mathcal{O}\left(\frac{\mu^{4}}{\Lambda_{\Sigma}^{2} f_{H}^{2}}\right)
$$

again consistent with the size of this correction for the toy linearly-realized auxiliary sector eq. (2.20). Higher-order terms in eq. (B.8) can also give $\mathcal{O}(1)$ shifts in the masses of the extra Higgs sector states $\Pi_{A}$. For example, the tadpole and first leading contribution to the masses of the $\Pi_{A}$ have the form

$$
\begin{aligned}
V\left(\Pi_{A}\right) & =\mu^{2} \Sigma \cdot H\left(1+c \frac{\mu^{2} \Sigma \cdot H}{\lambda_{\Sigma}^{2} f_{\Sigma}^{2}}\right)+\text { h.c. } \\
& \simeq \frac{1}{2} \mu^{2} \frac{v_{H}}{f_{\Sigma}}\left(1+2 c \frac{\mu^{2} v_{H}}{\Lambda_{\Sigma}^{2} f_{\Sigma}}\right) \Pi_{A}^{2}+\ldots
\end{aligned}
$$

Integrating out the $\Sigma$ sector also generates terms of the form $f\left(\frac{\mu^{2} v_{H}}{\Lambda_{\Sigma}^{2} f_{\Sigma}}\right)\left|D_{\mu}\langle\Sigma\rangle\right|^{2}$, which effectively shift the auxiliary EWSB vev from $f_{\Sigma}$ by an amount parametrically of the same size as the back-reaction in the linear sigma model, eq. (2.17).

For $\Lambda_{\Sigma} \simeq 4 \pi f_{\Sigma}$, back-reaction and higher-order terms result in $\lesssim \mathcal{O}(1)$ shifts to $f_{\Sigma}$ and $\alpha$, analogous to the results of the linear auxiliary sector analysis.

\section{B.2 Twin Higgs}

In the Twin model, the $H$ sector has an $\mathrm{SO}(8)_{H}$ global symmetry and the $\Sigma$ sector has a custodial $\mathrm{SO}(4)_{\Sigma_{A}} \times \mathrm{SO}(4)_{\Sigma_{B}} \times \mathbb{Z}_{2}$ global symmetry. The coupling of the $H$ and $\Sigma$ sector extends the form of the $\mathrm{SO}(5) / \mathrm{SO}(4)$ model, explicitly breaking the global symmetry to $\mathrm{SO}(4)_{A} \times \mathrm{SO}(4)_{B} \times \mathbb{Z}_{2}$,

$$
\mathcal{L} \supset \mathcal{O}_{\mu^{2}}=\hat{\mu}_{(A) I j}^{2} \mathcal{O}_{H}^{I} \mathcal{O}_{\Sigma_{A}}^{j}+\hat{\mu}_{(B) I j}^{2} \mathcal{O}_{H}^{I} \mathcal{O}_{\Sigma_{B}}^{j}
$$

Following the same strong coupling analysis as for the MCHM above, the IR contribution to the twin Higgs potential in the strongly coupled auxiliary sector regime has the generic form

$$
V_{\mu^{2}}(h)=\Lambda_{\Sigma}^{2} f_{\Sigma}^{2}\left[\hat{V}\left(\frac{\mu^{2} f_{H}}{\Lambda_{\Sigma}^{2} f_{\Sigma}} s_{h}\right)+\hat{V}\left(\frac{\mu^{2} f_{H}}{\Lambda_{\Sigma}^{2} f_{\Sigma}} c_{h}\right)\right]
$$

with the structure enforced by the $\mathbb{Z}_{2}$ symmetry. We choose to express the potential in terms of a redefined parameter $\mu^{2} \sim \hat{\mu}^{2}$ to normalize the tadpole term as $\mu_{I j}^{2} H_{A}^{I} \Sigma_{A}^{j}$.

As for the MCHM example, the higher-order terms are parametrically the same size as calculated in the linear realization for $\Lambda_{\Sigma} \simeq 4 \pi f_{\Sigma}$, such that the tadpole due to $f_{H}$ can readily constitute a significant perturbation on the $\Sigma_{B}$ sector. In addition, we expect the operators in the pNGB potential to be generated with $\mathcal{O}(1)$ coefficients, permitting the 
possibility that these terms can generate additional positive contributions to $\alpha$, perhaps alleviating the need for additional UV contributions required to overcome the $\delta \alpha<0$ from the SM top sector.

Another notable detail is that non-negligible higher-order terms coupling $H$ and $\Sigma$ should be generated. Depending on their sign and size, these terms may lead to complete breaking of $\mathrm{SU}(2)_{B} \times \mathrm{U}(1)_{B}$ (in the event that Twin hypercharge is gauged). In particular, as $f_{H} \gg v_{H}$ and $\left\langle\Sigma_{B}\right\rangle \sim\left\langle\Sigma_{A}\right\rangle$, higher-order terms can drive $\mathrm{SU}(2)$ alignment of $\left\langle H_{A}\right\rangle$ and $\left\langle\Sigma_{A}\right\rangle$ but misalignment of $\left\langle H_{B}\right\rangle$ and $\left\langle\Sigma_{B}\right\rangle$ even with $\mathbb{Z}_{2}$-symmetry. In this case, $\mathrm{SU}(2)_{B} \times \mathrm{U}(1)_{B}$ is fully broken while $\mathrm{SU}(2)_{A} \times \mathrm{U}(1)_{A} \rightarrow \mathrm{U}(1)_{\mathrm{EM}}$, avoiding a massless Twin hypercharge boson.

Open Access. This article is distributed under the terms of the Creative Commons Attribution License (CC-BY 4.0), which permits any use, distribution and reproduction in any medium, provided the original author(s) and source are credited.

\section{References}

[1] D.B. Kaplan and H. Georgi, $\mathrm{SU}(2) \times \mathrm{U}(1)$ breaking by vacuum misalignment, Phys. Lett. B 136 (1984) 183 [INSPIRE].

[2] D.B. Kaplan, H. Georgi and S. Dimopoulos, Composite Higgs scalars, Phys. Lett. B 136 (1984) 187 [INSPIRE].

[3] D.B. Kaplan, Flavor at SSC energies: a new mechanism for dynamically generated fermion masses, Nucl. Phys. B 365 (1991) 259 [inSPIRE].

[4] R. Contino and A. Pomarol, Holography for fermions, JHEP 11 (2004) 058 [hep-th/0406257] [INSPIRE].

[5] K. Agashe, R. Contino and A. Pomarol, The minimal composite Higgs model, Nucl. Phys. B 719 (2005) 165 [hep-ph/0412089] [InSPIRE].

[6] Z. Chacko, H.-S. Goh and R. Harnik, The twin Higgs: natural electroweak breaking from mirror symmetry, Phys. Rev. Lett. 96 (2006) 231802 [hep-ph/0506256] [INSPIRE].

[7] Z. Chacko, H.-S. Goh and R. Harnik, A twin Higgs model from left-right symmetry, JHEP 01 (2006) 108 [hep-ph/0512088] [INSPIRE].

[8] N. Arkani-Hamed et al., The minimal moose for a little Higgs, JHEP 08 (2002) 021 [hep-ph/0206020] [INSPIRE].

[9] N. Arkani-Hamed, A.G. Cohen, E. Katz and A.E. Nelson, The littlest Higgs, JHEP 07 (2002) 034 [hep-ph/0206021] [INSPIRE].

[10] I. Low, W. Skiba and D. Tucker-Smith, Little Higgses from an antisymmetric condensate, Phys. Rev. D 66 (2002) 072001 [hep-ph/0207243] [INSPIRE].

[11] D.E. Kaplan and M. Schmaltz, The little Higgs from a simple group, JHEP 10 (2003) 039 [hep-ph/0302049] [INSPIRE].

[12] ATLAS collaboration, Search for production of vector-like quark pairs and of four top quarks in the lepton-plus-jets final state in pp collisions at $\sqrt{s}=8 \mathrm{TeV}$ with the ATLAS detector, JHEP 08 (2015) 105 [arXiv: 1505.04306] [INSPIRE]. 
[13] CMS collaboration, Search for vector-like charge $2 / 3 T$ quarks in proton-proton collisions at $\sqrt{s}=8$ TeV, Phys. Rev. D 93 (2016) 012003 [arXiv:1509.04177] [InSPIRE].

[14] ATLAS collaboration, Search for single production of vector-like quarks decaying into Wb in pp collisions at $\sqrt{s}=8 \mathrm{TeV}$ with the ATLAS detector, Eur. Phys. J. C 76 (2016) 442 [arXiv: 1602.05606] [INSPIRE].

[15] A. Anandakrishnan, J.H. Collins, M. Farina, E. Kuflik and M. Perelstein, Odd top partners at the LHC, Phys. Rev. D 93 (2016) 075009 [arXiv: 1506.05130] [InSPIRE].

[16] J. Serra, Beyond the minimal top partner decay, JHEP 09 (2015) 176 [arXiv:1506.05110] [INSPIRE].

[17] ATLAS and CMS collaborations, Measurements of the Higgs boson production and decay rates and constraints on its couplings from a combined ATLAS and CMS analysis of the LHC pp collision data at $\sqrt{s}=7$ and $8 \mathrm{TeV}$, ATLAS-CONF-2015-044 (2015).

[18] CMS collaboration, Precise determination of the mass of the Higgs boson and tests of compatibility of its couplings with the standard model predictions using proton collisions at 7 and $8 \mathrm{TeV}$, Eur. Phys. J. C 75 (2015) 212 [arXiv:1412.8662] [inSPIRE].

[19] ATLAS collaboration, Measurements of the Higgs boson production and decay rates and coupling strengths using pp collision data at $\sqrt{s}=7$ and $8 \mathrm{TeV}$ in the ATLAS experiment, Eur. Phys. J. C 76 (2016) 6 [arXiv:1507.04548] [INSPIRE].

[20] B. Bellazzini, C. Csáki and J. Serra, Composite Higgses, Eur. Phys. J. C 74 (2014) 2766 [arXiv: 1401.2457] [INSPIRE].

[21] G. Panico and A. Wulzer, The composite Nambu-Goldstone Higgs, Lect. Notes Phys. 913 (2016) 1 [arXiv:1506.01961] [INSPIRE].

[22] S. Dawson et al., Working group report: Higgs boson, in Community Summer Study 2013: Snowmass on the Mississippi (CSS2013), Minneapolis U.S.A., 29 Jul-6 Aug 2013 [arXiv: 1310.8361] [INSPIRE].

[23] D.M. Asner et al., ILC Higgs white paper, in Community Summer Study 2013: Snowmass on the Mississippi (CSS2013), Minneapolis U.S.A., 29 Jul-6 Aug 2013 [arXiv:1310.0763] [INSPIRE].

[24] M.E. Peskin, Estimation of LHC and ILC capabilities for precision Higgs boson coupling measurements, in Community Summer Study 2013: Snowmass on the Mississippi (CSS2013), Minneapolis U.S.A., 29 Jul-6 Aug 2013 [arXiv:1312.4974] [INSPIRE].

[25] Z. Chacko, Y. Nomura, M. Papucci and G. Perez, Natural little hierarchy from a partially goldstone twin Higgs, JHEP 01 (2006) 126 [hep-ph/0510273] [INSPIRE].

[26] E.H. Simmons, Phenomenology of a technicolor model with heavy scalar doublet, Nucl. Phys. B 312 (1989) 253 [inSPIRE].

[27] S. Samuel, Bosonic technicolor, Nucl. Phys. B 347 (1990) 625 [inSPIRE].

[28] M. Dine, A. Kagan and S. Samuel, Naturalness in supersymmetry, or raising the supersymmetry breaking scale, Phys. Lett. B 243 (1990) 250 [INSPIRE].

[29] A. Kagan and S. Samuel, The family mass hierarchy problem in bosonic technicolor, Phys. Lett. B 252 (1990) 605 [INSPIRE].

[30] A. Kagan and S. Samuel, Renormalization group aspects of bosonic technicolor, Phys. Lett. B 270 (1991) 37 [INSPIRE].

[31] A. Kagan and S. Samuel, Bosonic technicolor in strings, Phys. Lett. B 284 (1992) 289 [INSPIRE]. 
[32] C.D. Carone and E.H. Simmons, Oblique corrections in technicolor with a scalar, Nucl. Phys. B 397 (1993) 591 [hep-ph/9207273] [INSPIRE].

[33] C.D. Carone and H. Georgi, Technicolor with a massless scalar doublet, Phys. Rev. D 49 (1994) 1427 [hep-ph/9308205] [INSPIRE].

[34] M. Antola, M. Heikinheimo, F. Sannino and K. Tuominen, Unnatural origin of fermion masses for technicolor, JHEP 03 (2010) 050 [arXiv:0910.3681] [INSPIRE].

[35] M. Antola, S. Di Chiara, F. Sannino and K. Tuominen, Supersymmetric extension of technicolor \& fermion mass generation, Nucl. Phys. B 864 (2012) 664 [arXiv:1111.1009] [INSPIRE].

[36] A.L. Kagan, Colored resonances from low scale bosonic technicolor, talk given at the 2008 KITP Workshop on Physics of the Large Hadron Collider, KITP, Santa Barbara U.S.A. (2008), http://online.itp.ucsb.edu/online/lhc08/kagan/.

[37] A.L. Kagan, Bosonic technicolor, or technicolor is alive and well, talk given at Naturalness 2014, Weizmann Institute, Rehovot Israel (2014), https://indico.cern.ch/event/290373/session/1/contribution/8.

[38] A.L. Kagan, The Higgs and vector resonances in bosonic technicolor, talk given at Physics from Run 2 of the LHC. 2nd NPKI Workshop, NPKI, Jeju Korea (2014), http://npki.org/.

[39] A. Azatov, J. Galloway and M.A. Luty, Superconformal technicolor, Phys. Rev. Lett. 108 (2012) 041802 [arXiv:1106.3346] [INSPIRE].

[40] A. Azatov, J. Galloway and M.A. Luty, Superconformal technicolor: models and phenomenology, Phys. Rev. D 85 (2012) 015018 [arXiv: 1106.4815] [INSPIRE].

[41] J. Galloway, M.A. Luty, Y. Tsai and Y. Zhao, Induced electroweak symmetry breaking and supersymmetric naturalness, Phys. Rev. D 89 (2014) 075003 [arXiv: 1306.6354] [INSPIRE].

[42] S. Chang, J. Galloway, M. Luty, E. Salvioni and Y. Tsai, Phenomenology of induced electroweak symmetry breaking, JHEP 03 (2015) 017 [arXiv:1411.6023] [INSPIRE].

[43] V. Sanz and J. Setford, Composite Higgses with seesaw EWSB, JHEP 12 (2015) 154 [arXiv: 1508.06133] [INSPIRE].

[44] G.C. Branco et al., Theory and phenomenology of two-Higgs-doublet models, Phys. Rept. 516 (2012) 1 [arXiv:1106.0034] [INSPIRE].

[45] R. Barbieri and A. Strumia, The 'LEP paradox', talk given at the 4th Rencontres du Vietnam: Physics at Extreme Energies (Particle Physics and Astrophysics), Hanoi Vietnam, 19-25 Jul 2000 [hep-ph/0007265] [INSPIRE].

[46] P. Batra, A. Delgado, D.E. Kaplan and T.M.P. Tait, The Higgs mass bound in gauge extensions of the minimal supersymmetric standard model, JHEP 02 (2004) 043 [hep-ph/0309149] [INSPIRE].

[47] R. Harnik, G.D. Kribs, D.T. Larson and H. Murayama, The minimal supersymmetric fat Higgs model, Phys. Rev. D 70 (2004) 015002 [hep-ph/0311349] [INSPIRE].

[48] L.J. Hall, D. Pinner and J.T. Ruderman, A natural SUSY Higgs near 126 GeV, JHEP 04 (2012) 131 [arXiv:1112.2703] [INSPIRE].

[49] E. Bertuzzo and C. Frugiuele, Natural SM-like 126 GeV Higgs boson via nondecoupling D terms, Phys. Rev. D 93 (2016) 035019 [arXiv:1412.2765] [InSPIRE].

[50] R. Barbieri, D. Greco, R. Rattazzi and A. Wulzer, The composite twin Higgs scenario, JHEP 08 (2015) 161 [arXiv: 1501.07803] [INSPIRE]. 
[51] M. Low, A. Tesi and L.-T. Wang, Twin Higgs mechanism and a composite Higgs boson, Phys. Rev. D 91 (2015) 095012 [arXiv: 1501.07890] [InSPIRE].

[52] R. Contino, T. Kramer, M. Son and R. Sundrum, Warped/composite phenomenology simplified, JHEP 05 (2007) 074 [hep-ph/0612180] [INSPIRE].

[53] G. Panico and A. Wulzer, The discrete composite Higgs model, JHEP 09 (2011) 135 [arXiv:1106.2719] [INSPIRE].

[54] M.E. Peskin, The alignment of the vacuum in theories of technicolor, Nucl. Phys. B 175 (1980) 197 [inSPIRE].

[55] M.A. Luty and T. Okui, Conformal technicolor, JHEP 09 (2006) 070 [hep-ph/0409274] [INSPIRE].

[56] M. Geller and O. Telem, Holographic twin Higgs model, Phys. Rev. Lett. 114 (2015) 191801 [arXiv: 1411.2974] [INSPIRE].

[57] C. Csáki, M. Geller, O. Telem and A. Weiler, The flavor of the composite twin Higgs, JHEP 09 (2016) 146 [arXiv: 1512.03427] [INSPIRE].

[58] H. Beauchesne, K. Earl and T. Grégoire, The spontaneous $\mathbb{Z}_{2}$ breaking twin Higgs, JHEP 01 (2016) 130 [arXiv:1510.06069] [INSPIRE].

[59] D.S.M. Alves, P.J. Fox and N.J. Weiner, Higgs signals in a type I 2HDM or with a sister Higgs, arXiv:1207.5499 [INSPIRE].

[60] G. Burdman, Z. Chacko, R. Harnik, L. de Lima and C.B. Verhaaren, Colorless top partners, a $125 \mathrm{GeV}$ Higgs and the limits on naturalness, Phys. Rev. D 91 (2015) 055007 [arXiv: 1411.3310] [INSPIRE].

[61] N. Craig, A. Katz, M. Strassler and R. Sundrum, Naturalness in the dark at the LHC, JHEP 07 (2015) 105 [arXiv: 1501.05310] [INSPIRE].

[62] D. Curtin and C.B. Verhaaren, Discovering uncolored naturalness in exotic Higgs decays, JHEP 12 (2015) 072 [arXiv: 1506.06141] [INSPIRE].

[63] C. Csáki, E. Kuflik, S. Lombardo and O. Slone, Searching for displaced Higgs boson decays, Phys. Rev. D 92 (2015) 073008 [arXiv: 1508.01522] [INSPIRE].

[64] CMS collaboration, Search for a pseudoscalar boson decaying into a $Z$ boson and the $125 \mathrm{GeV}$ Higgs boson in $\ell^{+} \ell^{-} b \bar{b}$ final states, Phys. Lett. B 748 (2015) 221 [arXiv: 1504.04710] [INSPIRE].

[65] C.D. Carone and M. Golden, Detecting the technirho in technicolor models with scalars, Phys. Rev. D 49 (1994) 6211 [hep-ph/9312303] [INSPIRE].

[66] ATLAS collaboration, Search for $W Z$ resonances in the fully leptonic channel using pp collisions at $\sqrt{s}=8 \mathrm{TeV}$ with the ATLAS detector, Phys. Lett. B 737 (2014) 223 [arXiv: 1406.4456] [INSPIRE].

[67] ATLAS collaboration, Search for vector-like B quarks in events with one isolated lepton, missing transverse momentum and jets at $\sqrt{s}=8 \mathrm{TeV}$ with the ATLAS detector, Phys. Rev. D 91 (2015) 112011 [arXiv: 1503.05425] [INSPIRE].

[68] CMS collaboration, Search for pair-produced vectorlike B quarks in proton-proton collisions at $\sqrt{s}=8 \mathrm{TeV}$, Phys. Rev. D 93 (2016) 112009 [arXiv:1507.07129] [INSPIRE].

[69] CMS collaboration, Search for top-quark partners with charge 5/3 in the same-sign dilepton final state, Phys. Rev. Lett. 112 (2014) 171801 [arXiv:1312.2391] [INSPIRE]. 
[70] K. Agashe, R. Contino, L. Da Rold and A. Pomarol, A custodial symmetry for Z $b \bar{b}$, Phys. Lett. B 641 (2006) 62 [hep-ph/0605341] [INSPIRE].

[71] J. Kearney, A. Pierce and J. Thaler, Exotic top partners and little Higgs, JHEP 10 (2013) 230 [arXiv:1306.4314] [INSPIRE].

[72] H.-C. Cheng, S. Jung, E. Salvioni and Y. Tsai, Exotic quarks in twin Higgs models, JHEP 03 (2016) 074 [arXiv: 1512.02647] [INSPIRE].

[73] K. Harigaya and Y. Nomura, A composite model for the $750 \mathrm{GeV}$ diphoton excess, JHEP 03 (2016) 091 [arXiv: 1602.01092] [INSPIRE].

[74] K. Harigaya and Y. Nomura, Composite models for the $750 \mathrm{GeV}$ diphoton excess, Phys. Lett. B 754 (2016) 151 [arXiv:1512.04850] [INSPIRE].

[75] Y. Nakai, R. Sato and K. Tobioka, Footprints of new strong dynamics via anomaly and the $750 \mathrm{GeV}$ diphoton, Phys. Rev. Lett. 116 (2016) 151802 [arXiv: 1512.04924] [InSPIRE].

[76] E. Molinaro, F. Sannino and N. Vignaroli, Minimal composite dynamics versus axion origin of the diphoton excess, Mod. Phys. Lett. A 31 (2016) 1650155 [arXiv:1512.05334] [InSPIRE].

[77] D. Buarque Franzosi and M.T. Frandsen, Symmetries and composite dynamics for the $750 \mathrm{GeV}$ diphoton excess, arXiv : 1601.05357 [INSPIRE].

[78] C.-W. Chiang, M. Ibe and T.T. Yanagida, Revisiting scalar quark hidden sector in light of 750-GeV diphoton resonance, JHEP 05 (2016) 084 [arXiv: 1512.08895] [INSPIRE].

[79] Y. Bai, J. Berger and R. Lu, $750 \mathrm{GeV}$ dark pion: cousin of a dark G-parity odd WIMP, Phys. Rev. D 93 (2016) 076009 [arXiv: 1512.05779] [INSPIRE].

[80] CMS collaboration, Search for high-mass diphoton resonances in pp collisions at $\sqrt{s}=8 \mathrm{TeV}$ with the CMS detector, CMS-PAS-EXO-12-045 (2015).

[81] ATLAS collaboration, Search for resonances decaying to photon pairs in 3.2 $\mathrm{fb}^{-1}$ of $\mathrm{pp}$ collisions at $\sqrt{s}=13 \mathrm{TeV}$ with the ATLAS detector, ATLAS-CONF-2015-081 (2015).

[82] J.M. No, V. Sanz and J. Setford, See-saw composite Higgs model at the LHC: linking naturalness to the $750 \mathrm{GeV}$ diphoton resonance, Phys. Rev. D 93 (2016) 095010 [arXiv: 1512.05700] [INSPIRE].

[83] M. Low, A. Tesi and L.-T. Wang, A pseudoscalar decaying to photon pairs in the early LHC Run 2 data, JHEP 03 (2016) 108 [arXiv: 1512.05328] [INSPIRE].

[84] A. Belyaev et al., Singlets in composite Higgs models in light of the LHC $750 \mathrm{GeV}$ diphoton excess, Phys. Rev. D 94 (2016) 015004 [arXiv: 1512.07242] [InSPIRE].

[85] B. Bellazzini, R. Franceschini, F. Sala and J. Serra, Goldstones in diphotons, JHEP 04 (2016) 072 [arXiv: 1512.05330] [INSPIRE].

[86] J. Galloway, A.L. Kagan and A. Martin, UV complete partially composite pseudo-Nambu Goldstone boson Higgs, Phys. Rev. D 95 (2017) 035038 [arXiv:1609.05883] [INSPIRE].

[87] S. Weinberg, Phenomenological Lagrangians, Physica A 96 (1979) 327 [InSPIRE].

[88] A. Manohar and H. Georgi, Chiral quarks and the nonrelativistic quark model, Nucl. Phys. B 234 (1984) 189 [inSPIRE].

[89] H. Georgi and L. Randall, Flavor conserving CP-violation in invisible axion models, Nucl. Phys. B 276 (1986) 241 [inSPIRE]. 